\title{
Associação entre sintomas depressivos e excesso de peso em adolescentes escolares: análise de inquéritos em 18 países
}

\author{
Dissertação apresentada à Faculdade de Medicina da \\ Universidade de São Paulo para obtenção do título de \\ Mestre em Ciências \\ Programa de: Medicina Preventiva \\ Orientador: Prof. Dr. Paulo Rossi Menezes
}

(Versão corrigida. Resolução CoPGr 6018/11, de 01 de novembro de 2011. A versão original está disponível na Biblioteca da FMUSP)

\section{São Paulo}

2016 


\title{
Associação entre sintomas depressivos e excesso de peso em adolescentes escolares: análise de inquéritos em 18 países
}

\author{
Dissertação apresentada à Faculdade de Medicina da \\ Universidade de São Paulo para obtenção do título de \\ Mestre em Ciências \\ Programa de: Medicina Preventiva \\ Orientador: Prof. Dr. Paulo Rossi Menezes
}

(Versão corrigida. Resolução CoPGr 6018/11, de 01 de novembro de 2011. A versão original está disponível na Biblioteca da FMUSP)

São Paulo

2016 
Dados Internacionais de Catalogação na Publicação (CIP)

Preparada pela Biblioteca da

Faculdade de Medicina da Universidade de São Paulo

Creprodução autorizada pelo autor

\section{Altoé, Natalia Motta}

Associação entre sintomas depressivos e excessos de peso em adolescentes escolares : análise de inquéritos em 18 países / Natalia Motta Altoé. -- São Paulo, 2016.

Dissertação(mestrado)--Faculdade de Medicina da Universidade de São Paulo. Programa de Medicina Preventiva.

Orientador: Paulo Rossi Menezes.

Descritores: 1.Adolescentes 2.Sintoma depressivos 3.Sobrepeso 4.Obesidade 5.Índice de massa corporal 6 .Estudos transversais 7.Epidemiologia 8.Saúde pública

USP/FM/DBD-93/16 


\section{Agradecimentos}

Sem ordem de importância ou proporção de contribuição na dissertação,

Agradeço aos colegas de pós-graduação Camila Monteiro, Andrea Tenório e Leandro Rezende com quem troquei informações e tirei dúvidas em relação à minha pesquisa e minhas análises.

Agradeço à Professora Renata Levy que me auxiliou com as análises de ponderação da amostra.

Agradeço ao Professor Wolney Conde pela contribuição em diversos aspectos do trabalho desde o início, incluindo principalmente as análises para estimativa de sobrepeso e obesidade.

Agradeço ao meu orientador Paulo Rossi Menezes por toda parceria e apoio durante o mestrado, tanto na produção da minha dissertação (nosso estudo), quanto para esclarecimentos de outras dúvidas e participação em outras atividades. 


\section{Agradecimento à FAPESP}

Agradeço a Fundação de Amparo à Pesquisa do Estado de São Paulo (FAPESP) que me concedeu bolsa de mestrado e reserva técnica para compra de materiais necessários na pesquisa. 


\section{Normalização Adotada}

Esta dissertação está de acordo com as seguintes normas, em vigor no momento desta publicação:

Referências: adaptado de International Committee of Medical Journals Editors (Vancouver).

Universidade de São Paulo. Faculdade de Medicina. Divisão de Biblioteca e Documentação. Guia de apresentação de dissertações, teses e monografias. Elaborado por Anneliese Carneiro da Cunha, Maria Julia de A. L. Freddi, Maria F. Crestana, Marinalva de Souza Aragão, Suely Campos Cardoso, Valéria Vilhena. 3a ed. São Paulo: Divisão de Biblioteca e Documentação; 2011.

Abreviaturas dos títulos dos periódicos de acordo com List of Journals Indexed in Index Medicus. 


\section{Resumo}

\section{SUMÁRIO}

Abstract

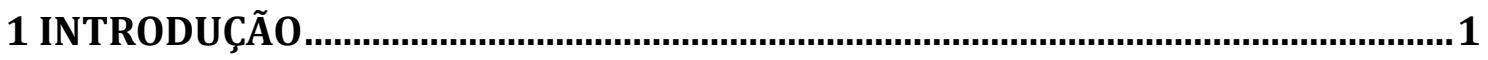

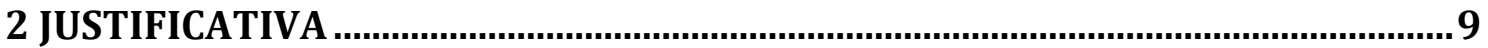

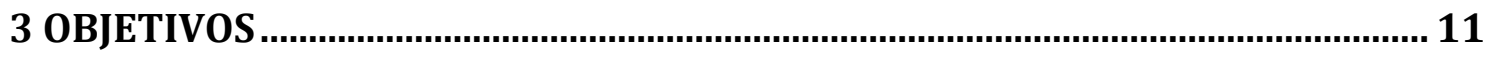

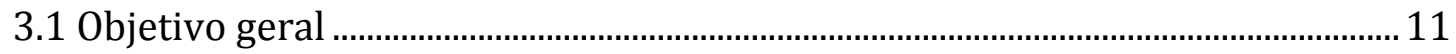

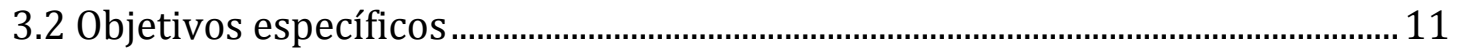

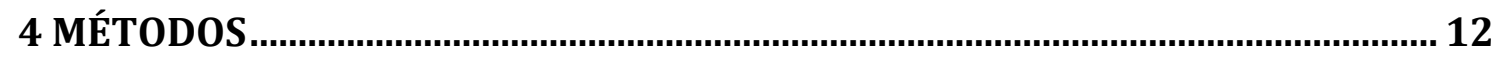

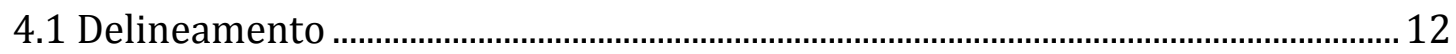

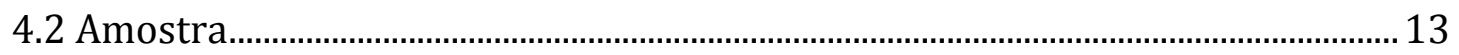

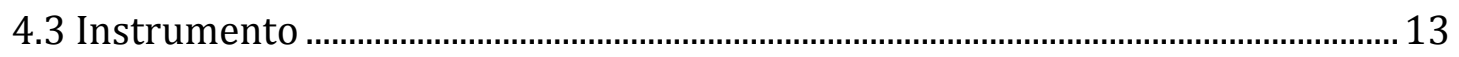

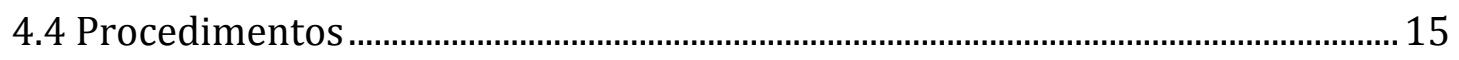

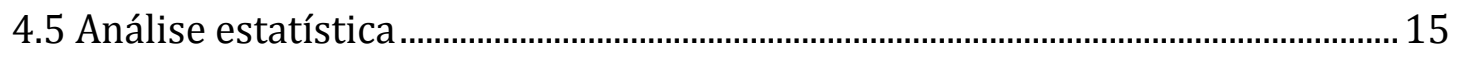

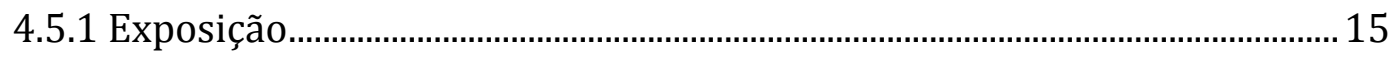

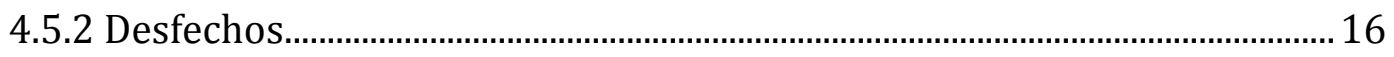

4.5.3 Variável modificadora de efeito …................................................................. 17

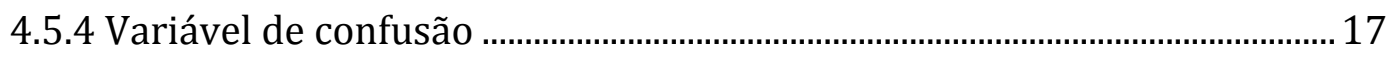

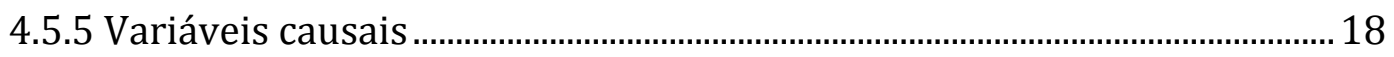

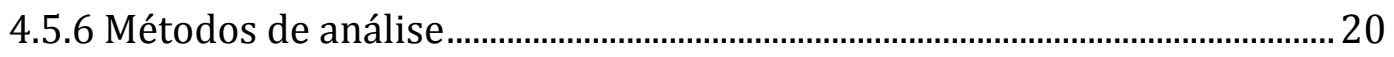

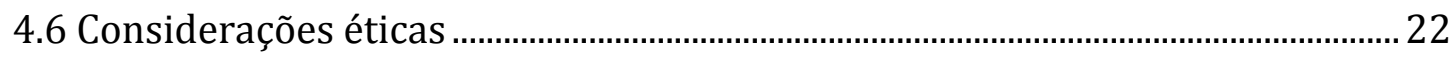

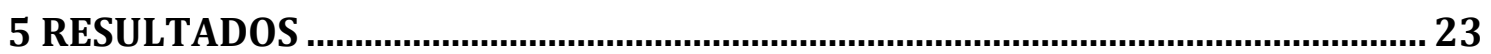

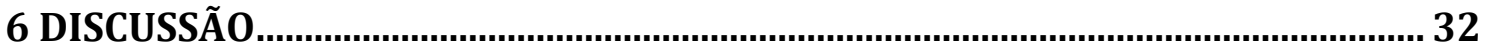

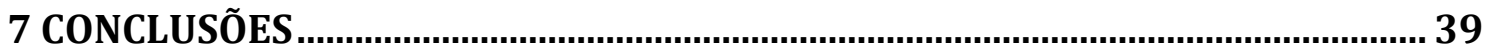

8 ANEXO

ANEXO A - Questionário principal da GSHS ….............................................................. 40

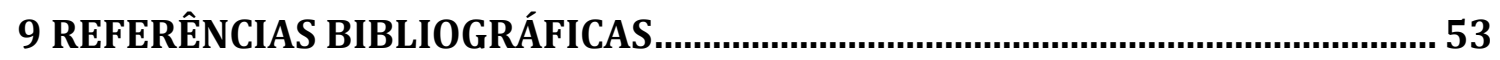




\section{RESUMO}

Altoé NM. Associação entre sintomas depressivos e excesso de peso em adolescentes escolares: análise de inquéritos em 18 países [dissertação]. São Paulo: Faculdade de Medicina, Universidade de São Paulo; 2016.

INTRODUÇÃO: Depressão e obesidade atualmente são considerados dois grandes problemas de saúde pública e têm elevado os custos em saúde mundialmente. Por causa de suas altas prevalências e do fato de que ambas aumentam o risco de doenças cardiovasculares, a associação entre elas vem sido investigada. 0 presente estudo tem por objetivo (a) estimar a prevalência de sintomas depressivos, sobrepeso e obesidade em adolescentes de diversos países, (b) ampliar conhecimentos prévios sobre a associação entre sintomas depressivos e sobrepeso/obesidade de países ou regiões específicas para um conjunto de países de diversas regiões, e (c) verificar se a associação varia de acordo com os diferentes contextos socioculturais. MÉTODO: Trata-se de um estudo transversal, onde analisamos dados de 18 países que participaram da Global School-based Student Health Survey (GSHS) entre 2003 e 2008. Nossa amostra foi de 88587 adolescentes (49,3\% do sexo masculino) com idades entre 11 e 17 anos. Calculamos o Índice de Massa Corporal (IMC) usando dados de peso e altura auto referidos. Classificamos sobrepeso e obesidade considerando valores de IMC de acordo com sexo e idade, conforme proposto por Cole \& Lobstein (2012). Estimamos sintomas depressivos a partir de questão auto referida do módulo de Saúde Mental do questionário da GSHS. Realizamos análises estatísticas para determinar (a) a prevalência de sintomas depressivos, sobrepeso e obesidade em cada país, (b) a associação entre sintomas depressivos e sobrepeso/obesidade em cada país, e (c) se existe heterogeneidade da associação entre os diferentes países. Os Odds Ratios (ORs) utilizados para estimar as associações foram obtidos através de análises de Mantel-Haenszel. RESULTADOS: Encontramos grande variação nas prevalências de sintomas depressivos (de 15,9\% no Myanmar a 49,6\% na Jordânia), sobrepeso (de 5,4\% no Sri Lanka a 40,0\% nos Emirados Árabes) e obesidade (de 0,8\% no Myanmar a 17,5\% nos Emirados Árabes). Quando estimamos os ORs para cada país, encontramos associação entre sintomas depressivos e sobrepeso/obesidade apenas na minoria dos países. Já nos dados da amostra total, composta por todos os adolescentes dos 18 países, encontramos associação de sintomas depressivos tanto com sobrepeso $(O R=1,20$; IC 95\%: 1,13$1,26)$, quanto com obesidade $(O R=1,26$; IC $95 \%: 1,15-1,38)$, apenas para o sexo feminino. CONCLUSÕES: Os resultados confirmam a associação entre sintomas depressivos e sobrepeso/obesidade em adolescentes do sexo feminino. 0 fato de a associação se mostrar homogênea entre os diferentes países, e de encontrarmos associação quando juntamos todos os países, pode sugerir que os contextos socioculturais específicos de cada país poderiam não exercer influência nessa associação. Estudos futuros que possam esclarecer melhor fatores causais e mediadores podem auxiliar na identificação e formulação de programas e políticas específicos para prevenção e tratamento de depressão e excesso de peso.

Descritores: adolescente; sintomas depressivos; sobrepeso; obesidade; índice de massa corporal; estudos transversais; epidemiologia; saúde pública. 


\begin{abstract}
Altoé NM. Association between depressive symptoms and overweight and obesity in scholar adolescents: analysis of surveys from 18 countries [dissertation]. São Paulo: "Faculdade de Medicina, Universidade de São Paulo"; 2016.

INTRODUCTION: Depression and obesity are currently considered important public health issues, causing large burden of disease and economic costs worldwide. Because of their high prevalence, and the fact that both are associated with increased risk for cardiovascular diseases, a possible association between them has been examined. The present study aims to (a) estimate the prevalence of depressive symptoms, overweight and obesity in adolescents from many different countries, (b) extend previous research on the association between depressive symptoms and overweight/obesity from specific country or region to several countries, and (c) verify if the association varies across the different sociocultural contexts. METHOD: Is a cross-sectional study were we analyzed data from 18 countries that participated of the Global School-based Student Health Survey (GSHS) between 2003 and 2008. Our sample contained 88587 adolescents (49.3\% males) aged 11 to 17 years. We calculated Body Mass Index (BMI) using selfreported weight and height. We classified overweight and obesity as proposed by Cole \& Lobstein (2012), considering BMI values according to the adolescent's age and sex. We assessed depressive symptoms with a self-reported question from the Mental Health module of the GSHS questionnaire. We conducted analyses to determine (a) the prevalence of depressive symptoms, overweight and obesity among the countries, (b) country-specific associations between depressive symptoms and overweight/obesity, and (c) if there was heterogeneity of the association across countries. RESULTS: There was marked cross-national variability in the prevalence of depressive symptoms (from 15.9\% in Myanmar to $49.6 \%$ in Jordan), overweight (from 5.4\% in Sri Lanka to $40.0 \%$ in the United Arab Emirates) and obesity (from $0.8 \%$ in Myanmar to $17.5 \%$ in the United Arab Emirates). Adjusting for age, eating behavior and physical activity, country-specific Odds Ratios (OR) for the association between depressive symptoms and overweight/obesity were only significant in a minority of countries. In pooled data across countries we found depressive symptoms significantly associated with overweight $(\mathrm{OR}=1.20 ; 95 \% \mathrm{CI}$ : 1.13-1.26) and obesity (OR $=1.26$; 95\% CI: 1.15 1.38), but only for females. CONCLUSIONS: These findings confirm the association between depressive symptoms and overweight/obesity among female adolescents. The fact that the association was homogeneous within the different countries and that we found association when examining pooled data across countries may suggest that country-specific sociocultural contexts could not make an influence on this association. Future studies that elucidate causal and mediating factors may help with identification and design of effective programs and policies for prevention and treatment of depression and overweight.
\end{abstract}

Descriptors: adolescent; depressive symptoms; overweight; obesity; body mass index; cross-sectional studies; epidemiology; public health. 



\section{INTRODUÇÃO}

\section{Depressão}

A depressão é uma doença psíquica que vem crescendo a cada ano (1) e que tornou-se atualmente uma grande preocupação para a saúde pública (2).

Diferente de flutuações usuais de humor e de respostas emocionais de curta duração aos desafios do dia-a-dia, especialmente quando tem longa duração e intensidade severa, a depressão pode se tornar um problema de saúde grave (1).

Existe atualmente uma estimativa de 350 milhões de pessoas afetadas com depressão em todo o mundo. 0 transtorno depressivo maior (major depressive disorder $(M D D)$ ) encontra-se entre as doenças com maior impacto para saúde pública em todo o mundo (3). Em casos mais graves, a depressão pode levar ao suicídio e atualmente é responsável por aproximadamente 1 milhão de mortes todos os anos (1). A depressão pode ocorrer em comorbidade com diversas outras condições crônicas de saúde $(4,5)$ e pode ser considerada responsável por aproximadamente $50 \%$ do aumento da mortalidade por diversas causas (6).

Segundo a Organização Mundial de Saúde (OMS) atualmente a depressão representa a principal causa de enfermidade e invalidez e o suicídio a terceira causa de morte entre adolescentes (7). 
No Brasil, segundo resultados da Pesquisa Nacional por Amostra de Domicílio (PNAD) realizada no país em 2008, 4,1\% dos entrevistados informaram estar com depressão (identificada por algum médico ou profissional de saúde) (8).

Um estudo recente realizado por Munhoz e col. em 2016 avaliou dados de 60202 indivíduos adultos que participaram da Pesquisa Nacional de Saúde (PNS) em 2013. Depressão foi rastreada usando-se o questionário Patient Health Questionnaire-9 (PHQ-9) que avaliou frequência de sintomas depressivos mais de duas semanas antes da coleta de dados. Novamente 4,1\% (IC 95\%: 3,8-4,4\%) dos brasileiros entrevistados foram identificados com depressão, mostrando que o problema encontrado em 2008 ainda persiste (9).

\section{Sobrepeso e obesidade}

A obesidade tem sido descrita como um importante problema de saúde pública da atualidade e vem ganhando destaque no cenário epidemiológico mundial. Assim como a depressão, a obesidade também está associada com aumento de mortalidade (10).

A prevalência mundial de obesidade mais que dobrou desde 1980, inclusive nos países em desenvolvimento como o Brasil, onde anteriormente predominavam os problemas relacionados à desnutrição (11).

Atualmente o sobrepeso e a obesidade constituem a quinta principal causa de morte no mundo. Aproximadamente 3,4 milhões de adultos morrem a cada ano em consequência de estarem acima do peso ou obesos. Estimativas da Organização Mundial de Saúde (OMS) apontam que até 2008 mais de 1.4 bilhões de adultos estavam com sobrepeso. Destes, 200 milhões de homens e 300 
milhões de mulheres entravam na faixa da obesidade, ou seja, mais de 1 em cada 10 adultos na população mundial estava obeso (11).

Os altos índices de obesidade não têm alcançado apenas a população adulta. Segundo a OMS em 2010 aproximadamente 35 milhões de crianças com sobrepeso viviam nos países em desenvolvimento e 8 milhões nos países desenvolvidos (11).

A Divisão de Pesquisas em Saúde e Nutrição dos Estados Unidos aponta que a obesidade em crianças de 6 a 11 anos no país triplicou entre 1976-1980 e 2007-2008, passando de 6.5\% para 19.6\% (12). Dados da Pesquisa Nacional de Verificação de Saúde e Nutrição (NHANES) de 2009-2010 revelaram que o problema persistia, pois $16,9 \%$ das crianças e adolescentes americanos continuavam obesos (13).

A infância e a fase inicial da adolescência representam etapas críticas para o desenvolvimento e crescimento saudáveis (14-16). A ocorrência de excesso de peso em idades cada vez mais precoces e o aumento das morbidades provocadas pela obesidade têm despertado a preocupação de pesquisadores e profissionais da área da saúde.

Assim como em adultos, a obesidade infantil é uma doença multissistêmica e está relacionada com o aparecimento de distúrbios cardiovasculares e endócrinos como hipertensão arterial, cardiopatias, diabetes, dislipidemias, dentre outros $(11,17-20)$.

Há também evidências de que crianças e adolescentes com valores do índice de massa corporal acima do esperado para a idade apresentam elevada probabilidade de conservar o excesso de peso na idade adulta (21). Segundo 
Serdula e col. (1993) um terço dos pré-escolares e metade dos escolares obesos se tornam adultos obesos (22).

Iniciativas de saúde pública atuais reconhecem a importância de se identificar as influências do excesso de peso na infância no peso do indivíduo quando adulto (23). Esta nova ênfase deriva da limitada disponibilidade de tratamentos eficazes para a obesidade, juntamente com a visão atual da obesidade como uma condição de desenvolvimento (24).

\section{$\underline{\text { Sobrepeso e obesidade no Brasil }}$}

Os resultados Pesquisa de Orçamentos Familiares (POF) realizada entre 2008 e 2009 encontraram que 20,5\% dos adolescentes brasileiros apresentava excesso de peso e 4,9\% obesidade. Nos últimos 35 anos a prevalência de excesso de peso entre os jovens aumentou aproximadamente seis vezes no sexo masculino (de $3,7 \%$ para $21,7 \%$ ) e quase três vezes no sexo feminino (de $7,6 \%$ para $19,4 \%)(25)$.

Um aspecto importante relacionado à obesidade diz respeito aos elevados custos que esse distúrbio e suas consequências representam para o sistema de saúde e para a sociedade. Os custos diretos com hospitalizações no Brasil indicam que os percentuais despendidos são similares aos de países desenvolvidos (26).

De acordo com o primeiro levantamento sobre os custos da obesidade realizado em 2003, aproximadamente 1 bilhão e 100 milhões de reais foram gastos a cada ano com internações hospitalares, consultas médicas e remédios para o tratamento do excesso de peso e suas doenças associadas. Apenas o Sistema Único de Saúde (SUS) destinou 600 milhões de reais para as internações 
por comorbidades relativas à obesidade. Esse valor equivaleu a $12 \%$ do que o governo brasileiro despendeu no ano com todas as outras doenças (27).

\section{Depressão e obesidade}

Como descrito anteriormente, depressão e obesidade atualmente são amplamente conhecidos como problemas maiores de saúde pública $(28,29)$ e vêm trazendo enorme aumento de custos com saúde $(1,30,31)$.

Ambos estão associados com várias doenças crônicas como diabetes, hipertensão, dislipidemia, câncer e doenças respiratórias e osteoarticulares (3235).

Obesidade poderia também ser uma explicação para ter quase dobrado o risco de doenças cardiovasculares, doenças cerebrovasculares e o excesso de mortalidade entre indivíduos com depressão $(6,11,36-38)$.

Por causa da alta prevalência de depressão e obesidade, e do fato de que ambas carregam um aumento do risco para doenças cardiovasculares, uma potencial associação entre elas vem sido repetidamente estudada.

\section{Estudos sobre a relação depressão-obesidade}

Em meio a inúmeros estudos que dão ênfase principalmente ao desequilíbrio do balanço energético e ao sedentarismo como causas de obesidade, e a doenças cardiovasculares e diabetes como consequências da obesidade, alguns estudos mais recentes vêm tentando associar o ganho de peso com a saúde mental. Esses estudos têm encontrando resultados positivos na correlação do peso com distúrbios mentais como a depressão, por exemplo. 
Já existe na literatura evidência convincente de que depressão e sintomas depressivos estão associados com excesso de peso, e vice-versa (39-47).

\section{Estudos transversais}

Diversos estudos transversais encontraram associação entre depressão ou sintomas depressivos e excesso de peso $(42,44,48-52)$.

Uma meta-análise de 17 estudos transversais (nove eram dos Estados Unidos da América (EUA)) relatou associação positiva entre depressão e obesidade. Eles encontraram um Odds Ratio $(O R)$ de 1,18, apenas para o sexo feminino. Idade e continente de residência foram utilizados como potenciais fatores de confusão, porém o valor de $O R$ ajustado à eles foi similar ao $O R$ bruto. $(42)$.

Estudos longitudinais

Um estudo prospectivo realizado com população adulta encontrou que depressão no baseline estava relacionada com ganho de peso no follow-up (53). Outros estudos prospectivos relataram evidências de uma associação bidirecional entre depressão e excesso de peso (54-56).

Uma meta-análise de 15 estudos longitudinais $(n=58745)$ encontrou evidência de associação bidirecional: (a) depressão aumentou em 58\% o risco de obesidade e (b) sobrepeso e obesidade aumentaram em $27 \%$ e $55 \%$, respectivamente, o risco de depressão. Vale observar que mais de $50 \%$ dos estudos incluídos haviam sido conduzidos nos EUA (56).

Por outro lado, uma revisão de 25 estudos prospectivos encontrou boa evidência da associação de obesidade-para-depressão, porém relatou evidências 
mistas de depressão-para-obesidade. Nessa revisão, depressão foi examinada como exposição para obesidade em 15 estudos e 8 deles (53\%) encontraram associação. Dentre os 15 estudos analisados, 11 deles (73,3\%) eram dos EUA, e em apenas 7 estudos (46,6\%) a amostra era composta por adolescentes no baseline (57).

Estudos longitudinais relacionando depressão durante infância e adolescência com desenvolvimento de obesidade na idade adulta

Franko e col. (2005) encontraram que participantes com níveis maiores de depressão na idade de 16 ou 18 anos tinham maior risco de obesidade com 21 $\operatorname{anos}(58)$.

Em um estudo de coorte, Goodman \& Whitaker (2002) acompanharam 9374 adolescentes durante 1 ano. Eles encontraram que adolescentes depressivos no baseline, comparados aos não depressivos, tiveram risco aumentado de desenvolvimento e persistência de obesidade durante a adolescência (39).

Hasler e col. (2005) mostraram que mulheres que tiveram depressão durante a infância, comparadas com as que não tiveram, possuíam maior risco de obesidade em idade adulta (59).

Pine e col. (2001) encontraram que crianças que tiveram depressão tinham maior índice de massa corporal (IMC) quando adultas do que aquelas que não tiveram depressão (40).

Richardson e col. (2003) relataram que meninas que tiveram depressão no fim da adolescência possuíam risco aumentado de desenvolver obesidade com 26 anos de idade (60). 
Vale ressaltar que a maioria dos estudos citados acima também foi conduzida com indivíduos norte americanos.

\section{Relação entre depressão e obesidade}

Pesquisadores levantaram alguns possíveis mecanismos pelos quais depressão e sintomas depressivos poderiam promover o ganho de peso.

Comer Emocional (Emotional Eating (EmE)) (61-64), hyperphagia (6567), inatividade física $(42,43,68,69)$, sono de má qualidade (70), e distúrbios neuroendócrinos (71-75), foram relacionados com depressão, sintomas depressivos ou depressão atípica.

Ainda, um padrão de sono ruim pode ser fator de risco também para obesidade (76), assim como algumas medicações psicotrópicas utilizadas no tratamento da depressão $(37,77-79)$. 


\section{JUSTIFICATIVA}

A alta prevalência de excesso de peso e de depressão, identificada através de inquéritos e estudos mundiais, mostra que ambos já constituem um problema de saúde pública no mundo .

A maior parte dos estudos que avaliaram sintomas depressivos e sua associação com excesso de peso entre adolescentes utilizaram dados de amostras representativas locais ou nacionais. A maior parte desses estudos foi conduzida nos continentes norte americano e europeu.

Embora diversos estudos já tenham investigado a associação entre depressão e obesidade, ainda existem algumas divergências nos resultados. Essas divergências podem ser indicativas da existência de uma influência de características socioculturais nesta associação.

Ao que sabemos, não existe ainda na literatura nenhum estudo avaliando a associação entre sintomas depressivos e excesso de peso em adolescentes de diversos países de diferentes continentes. Scott e col. (2008) estudaram essa associação investigando inquéritos transversais de diversos países, porém sua amostra era composta apenas por adultos (50).

Sendo assim, o presente estudo propõe-se a (a) estimar a prevalência de sintomas depressivos, sobrepeso e obesidade em adolescentes de diversos países, (b) estender pesquisas prévias sobre a relação depressão-obesidade de países e regiões específicos para diversos países (c) avaliar se essa associação varia de acordo com os diferentes contextos socioculturais presentes em cada país. 
Os resultados obtidos irão contribuir para quantificar a importância desta associação em adolescentes de diversas regiões do mundo e apresentar evidências que possam auxiliar no redirecionamento do foco da prevenção e do tratamento de ambas, depressão e obesidade. 


\section{OBJETIVOS}

\subsection{Objetivo geral}

Investigar a associação entre sintomas depressivos e excesso de peso em adolescentes de diversos países de diferentes continentes.

\subsection{Objetivos específicos}

- Investigar a frequência de sintomas depressivos e de excesso de peso em adolescentes de diversos países;

- Estimar a associação entre sintomas depressivos e o excesso de peso em adolescentes de diversos países;

- Investigar se há heterogeneidade da associação entre os diversos países; 


\section{MÉTODOS}

\subsection{Delineamento}

A Global School-based Student Health Survey (GSHS) está sendo desenvolvida pela Organização Mundial de Saúde (OMS) e pelo Centro de Controle e Prevenção de Doenças dos Estados Unidos (CDC) em parceria com UNICEF, UNESCO e UNAIDS, com objetivo de avaliar e comparar fatores comportamentais de risco e fatores protetivos relacionados às principais causas de morbidade e mortalidade no mundo (80).

A GSHS é uma pesquisa baseada na escola e trata-se de uma série de estudos transversais de base populacional, onde um questionário autoadministrado é aplicado a estudantes durante o período regular de aula (80).

A proposta da GSHS é fornecer dados sobre comportamentos de saúde e fatores de proteção entre estudantes almejando:

- Auxiliar os países no desenvolvimento de prioridades, no estabelecimento de programas e na defesa de recursos para programas e políticas de saúde do escolar e do adolescente;

- Permitir que as agências internacionais, os países e outros façam comparações entre os países quanto à prevalência de comportamentos de saúde e fatores de proteção;

- Estabelecer tendências na prevalência de comportamentos de saúde e fatores de proteção por país para utilizar na avaliação de programas de promoção de saúde e saúde do adolescente. 


\subsection{Amostra}

A GSHS é conduzida principalmente em estudantes com idade entre 13 e 15 anos, mas sua amostra total é composta por adolescentes de 11 a 17 anos de idade.

Um procedimento padronizado de amostragem é utilizado em cada local onde a pesquisa será realizada. Esse procedimento é composto por duas fases. Na primeira fase as unidades primárias de amostragem são as escolas, selecionadas com uma probabilidade proporcional ao seu tamanho de registro. 0 segundo passo é a seleção aleatória de salas de aula dentro de cada escola previamente selecionada. Nas salas de aula selecionadas, todos os alunos são convidados a participar da pesquisa (81).

0 inquérito está em processo de implementação em 114 países distribuídos entre África (24), América (34), Europa (3), Oriente Médio (20), Região Pacífico Ocidental (23) e Sudeste Asiático (10). Atualmente encontram-se disponíveis no site da GSHS bancos de dados de 83 países: 16 do continente africano, 27 do americano, 2 europeus, 17 do Oriente Médio, 15 da Região Pacífico Ocidental e 6 do Sudeste Asiático (81). 0 presente estudo utilizará uma amostra total de 88587 indivíduos de 18 diferentes países.

\subsection{Instrumento}

Cada país desenvolve seu questionário personalizado utilizando os seguintes componentes: módulos do questionário principal (ANEXO A), questões do questionário principal expandido e questões específicas do país. As questões são traduzidas na linguagem de instrução apropriada para os estudantes e um Piloto é realizado para testar a compreensão. Todas as questões compartilham 
características em comum para aumentar a fluidez da pesquisa e a compreensão dos estudantes.

Para auxiliar na proteção da privacidade dos estudantes, não são permitidas questões associadas a respostas condicionais, que induzem o respondente a pular aquelas que não dizem respeito à ele (81).

O Questionário Principal é dividido nos seguintes 10 módulos (80):

- Características demográficas;

- Atividade física;

- Comportamento alimentar;

- Comportamentos sexuais que contribuam para infecção por HIV, outras doenças sexualmente transmissíveis e gravidez indesejada;

- Fatores de proteção;

- Higiene;

- Saúde mental;

- Uso de álcool e outras drogas;

- Uso de tabaco;

- Violência e lesões não intencionais.

Pelo menos 6 módulos do questionário principal devem ser usados por cada país. Uma vez que um módulo é selecionado, todas as questões daquele módulo são utilizadas sem modificação. Cada módulo do Questionário Principal contém de 3 a 7 questões (81).

Os países podem optar por incluir ou não questões do Questionário Principal Expandido e questões específicas do país (81). 


\subsection{Procedimentos}

O questionário é administrado dentro da sala de aula durante o período regular de aula. A duração do período de aula é sujeita a variações de escola para escola e de país para país. Os estudantes completam o questionário voluntariamente e anonimamente, e suas respostas são registradas diretamente em folhas de respostas ópticas (81).

\subsection{Análise estatística}

Os bancos de dados da GSHS estão disponíveis no site da OMS e foram baixados via internet (81). Utilizamos o software Stata 12.0 para realizar todas análises estatísticas.

\subsubsection{Exposição}

Presença de sintomas depressivos foi medida a partir da seguinte pergunta do módulo de Saúde Mental do questionário principal:

1. Durante os últimos 12 meses, você já se sentiu tão triste e sem esperança quase todos os dias por duas semanas ou mais de um modo que você parou de realizar suas atividades usuais?

Nos bancos de dados a variável correspondente a essa questão é do tipo categórica binária, tendo como opções de resposta "sim" ou “não".

Vale ressaltar que essa pergunta por si só é insuficiente para mensurar a presença de sintomas depressivos. A utilizamos porque, devido a limitações do banco de dados, era a que tínhamos disponível. 


\subsubsection{Desfechos}

Utilizamos como desfechos as variáveis sobrepeso e obesidade. Estudos indicam que, pela fato da obesidade ser uma condição mais severa que o sobrepeso, ela estaria mais fortemente associada com desregulações biológicas e desfechos de saúde desfavoráveis (82, 83). Baseando-se nessa premissa, consideramos importante estimar a associação de sintomas depressivos com sobrepeso e com obesidade separadamente, e não com excesso de peso como um único desfecho.

\section{Peso e altura}

Dados de peso e altura dos estudantes foram obtidos através da resposta às seguintes questões do módulo de Comportamento Alimentar do questionário principal:

1. Qual é a sua altura sem estar calçado?

2. Quanto você pesa sem estar calçado?

As informações de peso (Kg) e altura (metros) estavam disponíveis nos bancos de dados nas formas de variável quantitativa discreta e quantitativa contínua, respectivamente.

\section{Índice de massa corporal (IMC)}

A partir dos valores de peso e altura calculamos o índice de massa corporal $\left(\mathrm{IMC}=\right.$ peso $\left.(\mathrm{kg}) / \operatorname{altura}^{2}(\mathrm{~m})\right)$ dos adolescentes. 


\section{Sobrepeso e obesidade}

Classificamos o estado nutricional conforme proposto por Cole \& Lobstein (2012) para classificação de excesso de peso em adolescentes de acordo com sexo e idade (84). Convertemos os IMCs em z escores e posteriormente classificamos com sobrepeso os indivíduos com z escores $>+1$ e $\leq+2$ e com obesidade os de $\mathrm{z}$ escore $>+2$.

\subsubsection{Variável modificadora de efeito}

Sexo

Estudos transversais prévios sugeriram que sexo atuou como modificador de efeito da relação entre depressão e obesidade (42, 50, 85, 86). Assim sendo, estratificamos por sexo todas as nossas análises.

A variável correspondente ao sexo é categórica binária. Foi obtida através da pergunta “Qual é o seu sexo?” ( 0 = masculino; 1 = feminino).

\subsubsection{Variável de confusão}

\section{Idade}

Controlamos em nossas análises a variável idade, que estava disponível no banco na forma categórica ordinal e foi acessada pela pergunta "Qual é a sua idade?”. Possuía como opções de resposta "11 anos de idade ou mais novo", "12 anos de idade", "13 anos de idade", "14 anos de idade", "15 anos de idade" e "16 anos de idade ou mais velho".

Para melhor visualizarmos a distribuição da amostra de acordo com a idade criamos uma variável com três categorias: 11 e 12 anos; de 13 a 15 anos; 16 e 17 anos de idade. 


\subsubsection{Variáveis causais}

\section{Comportamento alimentar}

As duas variáveis sobre comportamento alimentar disponíveis nos bancos de dados da GSHS eram:

1. Durante os últimos 30 dias, quantas vezes por dia você usualmente comeu frutas, como EXEMPLOS ESPECÍFICOS DO PAÍS?

2. Durante os últimos 30 dias, quantas vezes por dia você usualmente comeu vegetais, como EXEMPLOS ESPECÍFICOS DO PAÍS?

Ambas variáveis são categóricas ordinais e possuem as seguintes opções de resposta: eu não comi frutas/vegetais durante os últimos 30 dias; menos de uma vez por dia; 1 vez por dia; 2 vezes por dia; 3 vezes por dia; 4 vezes por dia; 5 ou mais vezes por dia.

Dentre características encontradas em indivíduos com sintomas depressivos estão o comer emocional $(E m E)(61,62)$, a hiperfagia (65-67) e o aumento do consumo de alimentos doces e de maior densidade calórica (63). Pesquisadores sugerem que esses poderiam ser alguns dos fatores que explicariam a associação entre sintomas depressivos e a adiposidade.

Considerando que a hiperfagia e o comer emocional costumam estar relacionados com a adoção de preferências alimentares não saudáveis (42, 43, 68, 69), e que as nossas variáveis de comportamento alimentar avaliam proxy de alimentação saudável, as consideramos como variáveis causais. Isso porque, nessa linha de raciocínio, o desenvolvimento de um comportamento alimentar não saudável seria adotado por indívíduos com sintomas depressivos, o que poderia levar ao ganho de peso, estando no meio da cadeia causal. 


\section{Atividade física}

Estudos apontam que a inatividade física em adultos está associada tanto com transtorno depressivos quanto com IMC não eutrófico $(42,43,68,69)$. Ainda, a diminuição da prática de atividade física é considerada como um dos sintomas de depressão no Manual Estatístico de Diagnóstico de Desordens Mentais, quarta edição (Diagnostic and Statistical Manual of Mental Disorders (DSM-IV)). Sendo assim, inatividade física potencialmente estaria no caminho causal que une depressão ao ganho de peso e por isso não foi considerada como condundidora em nosso estudo.

Havia no banco de dados da GSHS duas questões, uma (1) indicativa de atividade física e outra (2) de comportamento sedentário:

1. Durante uma semana típica ou usual, em quantos dias você é fisicamente ativo por um total de pelo menos 60 minutos por dia?

2. Quanto tempo você gasta durante um dia típico ou usual sentando e assistindo televisão, jogando jogos de computador, conversando com amigos, ou fazendo outras atividades sentado, como EXEMPLOS ESPECÍFICOS DO PAÍS?

As variáveis correspondentes às duas perguntas estavam apresentadas na forma categórica ordinal.

A primeira questão, sobre atividade física, tem como opções de respostas as seguintes categorias: 0 dias; 1 dia; 2 dias; 3 dias; 4 dias; 5 dias; 6 dias; e 7 dias.

A segunda, sobre comportamento sedentário, tem as seguintes: menos de 1 hora por dia; 1 a 2 horas por dia; 3 a 4 horas por dia; 5 a 6 horas por dia; 7 a 8 horas por dia; mais que 8 horas por dia. 


\subsubsection{Métodos de análise}

Utilizamos em nossas análises os bancos de dados de países que participaram da pesquisa da GSHS entre 2003 e 2008, pois somente eles continham todas as principais variáveis de interesse para o nosso estudo.

Fizemos uma análise descritiva das prevalências de observações nas variáveis de exposição (sintomas depressivos), desfechos (peso e altura, que posteriormente foram utilizados para classificação de sobrepeso e obesidade), modificadora de efeito (sexo) e controle (idade).

Dentre os bancos previamente selecionados, incluímos em nosso estudo os que obtiveram $50 \%$ ou mais de respostas nas variáveis de interesse. Sendo assim, este estudo analisou dados de inquéritos da GSHS de 18 países, realizados nos período entre 2003 e 2008 ( $\mathrm{n}=88587$ adolescentes).

\section{Análise descritiva}

Fizemos uma análise descritiva com objetivo de conhecer as características demográficas da amostra por país, bem como as prevalências de sintomas depressivos, sobrepeso e obesidade em cada país.

\section{Peso da amostra complexa}

Consideramos o peso da amostra complexa para realizarmos todas as análises descritivas e de prevalências. Utilizamos o comando survey (svyset psu [pweight=weight], strata(stratum)) do STATA. As três seguintes variáveis encontravam-se disponíveis nos bancos da GSHS: (a) weight - permite que os resultados da GSHS sejam generalizados para todos os estudantes com características demográficas similares, e não apenas os que participaram da pesquisa; (b) stratum - reflete o primeiro nível do processo de amostragem da 
GSHS, que foi a escola. Cada stratum contem uma ou duas escolas, dependendo do tamanho da escola; (c) PSU - reflete o segundo nível do processo de amostragem da GSHS, que foram as salas de aula. Cada PSU é composto por uma ou mais salas de aula dentro de uma escola.

\section{Análises de associação}

Para uma primeira análise da relação entre os sintomas depressivos e o excesso de peso, e de como esta relação se comporta nos 18 diferentes países, foram estimadas as prevalências de sobrepeso e de obesidade em função dos sintomas depressivos em cada país.

Para estimarmos a associação entre sintomas depressivos e sobrepeso e sintomas depressivos e obesidade, calculamos Odds Ratios (ORs) brutos (Modelo 1) e ORs ajustados para idade (Modelo 2). Os ORs foram estimados por meio de análises de Mantel-Haenszel (mhodds). Consideramos valores de $p<0,05$ e intervalos de 95\% de confiança (IC95\%).

\section{Teste de Homogeneidade (TH)}

Para verificarmos a existência de heterogeneidade da associação entre sintomas depressivos e sobrepeso e obesidade entre os diversos países, utilizamos os resultados do Teste de Homogeneidade (Test of Homogeneity - TH). Consideramos como heterogêneas as associações que obtivessem valores de $p<0,05$ no $T H$, ou seja, se o valor de $p$ fosse $>0,05$ poderíamos considerar que não havia heterogeneidade entre os países no que diz respeito à associação buscada. 
Os resultados que indicaram homogeneidade nos possibilitaram estimar a associação entre desfecho e exposição para a amostra total, composta por todos os adolescentes de todos os 18 países.

\section{Medida de associação}

De acordo com Coutinho e col. (2008) (87), valores de ORs não devem ser interpretados como Razões de Prevalência (RPs) quando a prevalência do desfecho é intermediária ou alta. Como a prevalência de sobrepeso é alta em alguns países, decidimos estimar a Razão de Prevalência (RP) da associação entre sintomas depressivos e sobrepeso e obesidade. Calculamos as RPs através da Regressão de Poisson. Como os valores de ORs e de RPs foram muito próximos, optamos por apresentar nossos resultados em valores de ORs.

\subsection{Considerações éticas}

Relatórios disponibilizados pelos países onde a $G S H S$ já foi implementada declaram que, em relação às considerações éticas, foram obtidas permissões para realização do estudo dos Ministérios de Saúde e Educação do país. Foi recolhido dos diretores das escolas e dos responsáveis dos alunos um Consentimento Livre e Esclarecido para participação no estudo. A confidencialidade foi mantida através da permissão de participação anônima e voluntária conforme as exigências de ética em pesquisa.

O projeto dessa pesquisa, sob o número de Protocolo de Pesquisa 467/13, foi aprovado em 27 de novembro de 2013 pela Comissão de Ética em Pesquisa da Faculdade de Medicina da USP (CEP - FMUSP). 


\section{RESULTADOS}

\section{Características da amostra}

As características da amostra distribuídas de acordo com sexo e idade podem ser observadas na Tabela 1.

\section{Características da amostra total - (Tabela 1)}

Os sexos feminino e masculino estavam distribuídos de forma similar na amostra total (49,3\% adolescentes do sexo masculino).

Com relação à idade observamos que, conforme o propósito da pesquisa, a maior parte dos adolescentes estava na faixa etária entre 13 e 15 anos de idade $(75,7 \%)$.

Características das amostras em cada país - (Tabela 1)

As prevalências mínima e máxima de adolescentes do sexo masculino foram encontradas no Uruguai $(45,2 \%)$ e na República do Jibuti (Djibouti) $(60,2 \%)$

A prevalência de adolescentes com idade entre 13 e 15 anos variou de 52,7\% na República do Jibuti (Djibouti) a 93,5\% na Indonésia. 


\begin{tabular}{|c|c|c|c|c|c|c|c|c|c|c|}
\hline \multirow{2}{*}{ NUM } & \multirow{2}{*}{ REGIÃO } & \multirow{2}{*}{ PAÍS } & \multirow{2}{*}{ ANO } & \multirow{2}{*}{ AMOSTRA } & \multirow{2}{*}{$\mathbf{N}$} & \multicolumn{2}{|c|}{ Sexo } & \multicolumn{3}{|c|}{ Idade } \\
\hline & & & & & & $\mathbf{M}$ & $\mathbf{F}$ & $\begin{array}{c}11-12 \\
\text { anos }\end{array}$ & $\begin{array}{c}13-15 \\
\text { anos }\end{array}$ & $\begin{array}{c}16-17 \\
\text { anos }\end{array}$ \\
\hline 1 & África & Seychelles & 2007 & nacional & 1417 & 49,7 & 50,3 & 18,3 & 60,4 & 21,4 \\
\hline 2 & África & Uganda & 2003 & nacional & 3142 & 51,2 & 48,8 & 1,8 & 60,5 & 37,8 \\
\hline 3 & América & Argentina & 2007 & nacional & 1951 & 48,0 & 52,0 & 1,8 & 73,4 & 24,8 \\
\hline 4 & América & $\begin{array}{l}\text { Cayman } \\
\text { Islands }\end{array}$ & 2007 & nacional & 1291 & 51,5 & 48,5 & 15,1 & 73,3 & 11,6 \\
\hline 5 & América & Chile & $\begin{array}{c}2004 \\
2005^{1}\end{array}$ & $\begin{array}{l}\text { Região } \\
\text { Metropolitana }^{1}\end{array}$ & 5633 & 51,5 & 48,5 & 14,3 & 79,2 & 6,6 \\
\hline 6 & América & Ecuador & 2007 & Capital - Quito ${ }^{2}$ & 2194 & 51,5 & 48,5 & 31,0 & 60,2 & 8,9 \\
\hline 7 & América & Uruguay & 2006 & nacional & 3393 & 45,2 & 54,8 & 11,0 & 70,4 & 18,6 \\
\hline 8 & $\begin{array}{l}\text { Sudeste } \\
\text { Asiático }\end{array}$ & India & 2007 & nacional & 8049 & 58,1 & 41,9 & 8,1 & 84,5 & 7,4 \\
\hline 9 & $\begin{array}{l}\text { Sudeste } \\
\text { Asiático }\end{array}$ & Indonesia & 2007 & nacional & 3103 & 49,9 & 50,1 & 3,9 & 93,5 & 2,7 \\
\hline 10 & $\begin{array}{l}\text { Sudeste } \\
\text { Asiático }\end{array}$ & Myanmar & 2007 & nacional & 2804 & 50,8 & 49,2 & 13,4 & 73,4 & 13,2 \\
\hline 11 & $\begin{array}{l}\text { Sudeste } \\
\text { Asiático }\end{array}$ & Sri lanka & 2008 & nacional & 2591 & 50,0 & 50,0 & 9,6 & 87,1 & 3,2 \\
\hline 12 & $\begin{array}{l}\text { Sudeste } \\
\text { Asiático }\end{array}$ & Thailand & 2008 & nacional & 2761 & 48,5 & 51,5 & 17,0 & 79,3 & 3,7 \\
\hline 13 & $\begin{array}{l}\text { Oriente } \\
\text { Médio }\end{array}$ & Djibouti & 2007 & nacional & 1774 & 60,2 & 39,8 & 4,1 & 52,7 & 43,2 \\
\hline 14 & $\begin{array}{l}\text { Oriente } \\
\text { Médio }\end{array}$ & Egypt & 2006 & nacional & 5189 & 51,9 & 48,2 & 22,3 & 75,4 & 2,3 \\
\hline 15 & $\begin{array}{l}\text { Oriente } \\
\text { Médio }\end{array}$ & Jordan & $\begin{array}{l}2004 \\
2007\end{array}$ & nacional & 4604 & 50,4 & 49,6 & 0,9 & 74,0 & 25,2 \\
\hline 16 & $\begin{array}{l}\text { Oriente } \\
\text { Médio }\end{array}$ & $\begin{array}{l}\text { United Arab } \\
\text { Emirates }\end{array}$ & 2005 & nacional & 15634 & 50,0 & 50,0 & 15,8 & 67,1 & 17,2 \\
\hline 17 & $\begin{array}{l}\text { Pacífico } \\
\text { Ocidental }\end{array}$ & China & 2003 & 4 Regiões $^{3}$ & 8893 & 51,2 & 48,8 & 13,8 & 82,1 & 4,1 \\
\hline 18 & $\begin{array}{l}\text { Pacífico } \\
\text { Ocidental }\end{array}$ & Philippines & $\begin{array}{l}2003 \\
2007\end{array}$ & nacional & 12921 & 45,5 & 54,6 & 1,2 & 64,6 & 34,3 \\
\hline TOTA & & & & & 88587 & 49,3 & 50,7 & 8,3 & 75,7 & 16,0 \\
\hline
\end{tabular}

FONTE: Global School-based Student Health Survey (GSHS) - WHO.

1 Os dados do Chile correspondem a três etapas de pesquisa, uma em 2004 e duas fases em 2005. A amostra, nas três fases da pesquisa, representa a região metropolitana do Chile.

2 No Ecuador a amostra representa sua capital Quito.

$3 \mathrm{Na}$ China a pesquisa foi realizada em quatro regiões diferentes: Beijing, Hangzhou, Wuhan e Wurumqi. Sua amostra total neste estudo foi composta pela união dessas quatro regiões. 


\section{Prevalências de sintomas depressivos, sobrepeso e obesidade}

\section{Prevalências na amostra total}

Para amostra total, a prevalência de sintomas depressivos foi maior dentre meninas $(32,0 \%)$, enquanto que meninos tiveram maiores prevalências de sobrepeso $(18,9 \%)$ e de obesidade $(5,9 \%)$ (Tabela 2$)$.

Tabela 2 - Prevalências (\%) ponderadas de sintomas depressivos, sobrepeso e obesidade, distribuídas por sexo - 18 países - 2003 a 2008

\begin{tabular}{|c|c|c|c|c|c|c|c|c|c|}
\hline \multirow{2}{*}{ PAÍS } & \multicolumn{3}{|c|}{ SINTOMAS DEPRESSIVOS } & \multicolumn{3}{|c|}{ SOBREPESO } & \multicolumn{3}{|c|}{ OBESIDADE } \\
\hline & $\mathbf{M}$ & $\mathbf{F}$ & Total & $\mathbf{M}$ & $\mathbf{F}$ & Total & $\mathbf{M}$ & $\mathbf{F}$ & Total \\
\hline Seychelles & 27,9 & 35,4 & 31,7 & 24,0 & 27,6 & 25,9 & 10,1 & 11,4 & 10,8 \\
\hline Uganda & 40,2 & 44,0 & 42,1 & 2,7 & 14,7 & 8,6 & 0,5 & 1,4 & 0,9 \\
\hline Argentina & 26,1 & 35,4 & 31,0 & 31,6 & 18,7 & 25,1 & 6,5 & 3,1 & 4,7 \\
\hline Cayman Islands & 21,2 & 29,3 & 25,1 & 40,9 & 31,8 & 36,7 & 16,4 & 11,7 & 14,2 \\
\hline Chile & 21,3 & 40,8 & 30,8 & 39,3 & 32,8 & 36,1 & 11,1 & 7,9 & 9,5 \\
\hline Ecuador & 23,0 & 31,4 & 27,0 & 37,8 & 32,4 & 35,1 & 11,9 & 8,1 & 10,0 \\
\hline Uruguay & 12,4 & 22,1 & 17,8 & 31,6 & 19,7 & 25,2 & 5,5 & 3,1 & 4,2 \\
\hline India & 26,1 & 24,8 & 25,5 & 13,8 & 10,7 & 12,5 & 3,2 & 1,8 & 2,6 \\
\hline Indonesia & 20,6 & 22,1 & 21,4 & 16,6 & 7,8 & 12,0 & 3,2 & 1,4 & 2,2 \\
\hline Myanmar & 17,0 & 14,7 & 15,9 & 5,8 & 6,5 & 6,2 & 1,1 & 0,6 & 0,8 \\
\hline Sri lanka & 34,0 & 31,0 & 32,5 & 5,3 & 5,6 & 5,4 & 0,7 & 1,1 & 0,9 \\
\hline Thailand & 18,2 & 15,1 & 16,6 & 24,4 & 15,6 & 19,8 & 8,9 & 5,4 & 7,1 \\
\hline Djibouti & 40,0 & 41,5 & 40,6 & 13,3 & 24,4 & 17,7 & 3,5 & 6,7 & 4,8 \\
\hline Egypt & 32,0 & 38,3 & 35,0 & 29,4 & 32,9 & 31,2 & 9,7 & 9,0 & 9,4 \\
\hline Jordan & 48,3 & 51,1 & 49,6 & 27,5 & 16,6 & 22,4 & 7,2 & 3,3 & 5,4 \\
\hline United Arab Emirates & 32,0 & 38,5 & 35,3 & 41,2 & 38,8 & 40,0 & 19,4 & 15,6 & 17,5 \\
\hline China & 18,9 & 19,1 & 19,5 & 23,1 & 13,7 & 18,4 & 8,4 & 3,8 & 6,1 \\
\hline Philippines & 38,2 & 42,7 & 40,7 & 13,9 & 10,4 & 12,0 & 5,9 & 3,8 & 4,8 \\
\hline TOTAL & 28,6 & 32,0 & 30,3 & 18,9 & 14,6 & 16,7 & 5,9 & 4,0 & 4,9 \\
\hline
\end{tabular}

FONTE: Global School-based Student Health Survey (GSHS) - WHO. 
Prevalências em cada país

Ainda na Tabela 2 podemos observar uma grande variação entre os países no que diz respeito às prevalências de sintomas depressivos (de 15,9\% em Myanmar a 49,6\% na Jordânia), sobrepeso (de 5,4\% no Sri Lanka a 40,0\% nos Emirados Árabes) e obesidade (de 0,8\% em Myanmar a 17,5\% nos Emirados Árabes).

Quando distribuídas por sexo, encontramos também grande variação nas prevalências de sintomas depressivos, sobrepeso e obesidade (Tabela 2).

\section{Associação entre sintomas depressivos e sobrepeso em cada país}

$\underline{\text { Sexo masculino }}$

A Tabela 3 apresenta a associação entre sintomas depressivos e sobrepeso para o sexo masculino, de acordo com valores de Odds Ratios (ORs) brutos (Modelo 1) e ORs ajustados por idade (Modelo 2). Estão apresentados também os valores dos intervalos de 95\% de confiança (IC 95\%) e seus respectivos valores de $p$. 
Tabela 3 - Associação entre sintomas depressivos e sobrepeso em adolescentes do sexo masculino - 18 países - 2003 a 2008

\begin{tabular}{|c|c|c|c|c|c|c|c|c|}
\hline \multirow{3}{*}{$\begin{array}{l}\text { PAÍS } \\
\text { Seychelles }\end{array}$} & \multicolumn{4}{|c|}{ Modelo 1} & \multicolumn{4}{|c|}{ Modelo 2} \\
\hline & \multirow{2}{*}{$\frac{O R}{0,77}$} & \multicolumn{2}{|c|}{ IC 95\% } & \multirow{2}{*}{$\begin{array}{c}\boldsymbol{p} \\
0,329\end{array}$} & \multirow{2}{*}{$\frac{\boldsymbol{O R}}{0,74}$} & \multicolumn{2}{|c|}{ IC 95\% } & \multirow{2}{*}{$\begin{array}{c}\boldsymbol{p} \\
0,249\end{array}$} \\
\hline & & 0,46 & - 1,29 & & & 0,44 & - 1,24 & \\
\hline Uganda & 1,99 & 1,08 & $-3,67$ & 0,024 & 2,11 & 1,10 & $-4,02$ & 0,021 \\
\hline Argentina & 0,88 & 0,60 & - 1,28 & 0,498 & 0,95 & 0,65 & - 1,40 & 0,796 \\
\hline Cayman Islands & 1,32 & 0,79 & $-2,21$ & 0,289 & 1,31 & 0,78 & $-\quad 2,19$ & 0,310 \\
\hline Chile & 1,00 & 0,82 & - 1,23 & 0,981 & 1,04 & 0,85 & - 1,28 & 0,723 \\
\hline Ecuador & 1,03 & 0,74 & - 1,42 & 0,875 & 1,08 & 0,78 & $-1,50$ & 0,641 \\
\hline Uruguay & 1,32 & 0,92 & - 1,88 & 0,125 & 1,39 & 0,98 & - 1,98 & 0,062 \\
\hline India & 0,97 & 0,77 & - 1,22 & 0,791 & 1,00 & 0,79 & - 1,27 & 0,963 \\
\hline Indonesia & 1,30 & 0,90 & - 1,86 & 0,161 & 1,32 & 0,92 & - 1,91 & 0,128 \\
\hline Myanmar & 0,89 & 0,46 & - 1,73 & 0,734 & 0,90 & 0,47 & - 1,74 & 0,756 \\
\hline Sri lanka & 0,63 & 0,32 & - 1,22 & 0,165 & 0,64 & 0,33 & - 1,24 & 0,181 \\
\hline Thailand & 0,99 & 0,71 & - 1,39 & 0,961 & 1,00 & 0,71 & - 1,42 & 0,975 \\
\hline Djibouti & 0,65 & 0,43 & - 0,99 & 0,043 & 0,68 & 0,44 & - 1,05 & 0,082 \\
\hline Egypt & 1,04 & 0,86 & - 1,27 & 0,671 & 1,08 & 0,88 & - 1,32 & 0,457 \\
\hline Jordan & 0,96 & 0,76 & - 1,21 & 0,706 & 0,97 & 0,77 & - 1,22 & 0,766 \\
\hline United Arab Emirates & 0,97 & 0,88 & - 1,08 & 0,621 & 1,02 & 0,92 & - 1,13 & 0,722 \\
\hline China & 1,00 & 0,82 & $-1,22$ & 0,984 & 1,04 & 0,85 & - 1,27 & 0,716 \\
\hline Philippines & 0,82 & 0,68 & - 0,99 & 0,033 & 0,84 & 0,70 & - 1,01 & 0,063 \\
\hline Test of Homogeneity = & & & & 22,57 & & & & 22,97 \\
\hline Pr $>$ chi2 = & & & & 0,163 & & & & 0,150 \\
\hline
\end{tabular}

FONTE: Global School-based Student Health Survey (GSHS) - WHO.

Controlando para idade encontramos associação positiva entre sintomas depressivos e sobrepeso em meninos de Uganda (OR = 2,11; IC 95\%: 1,10-4,02) (Tabela 03, Modelo 2).

$\underline{\text { Sexo feminino }}$

A Tabela 4 nos mostra que os ORs ajustados (Modelo 2) da estimativa da associação entre sintomas depressivos e sobrepeso para o sexo feminino foram 
significantes apenas em meninas das Ilhas Cayman $(O R=2,05$; IC 95\%: 1,22$3,45)$ e do Chile (OR = 1,28; IC 95\%: 1,08-1,51).

Tabela 4 - Associação entre sintomas depressivos e sobrepeso em adolescentes do sexo feminino - 18 países - 2003 a 2008

\begin{tabular}{|c|c|c|c|c|c|c|c|c|}
\hline \multirow{3}{*}{$\begin{array}{l}\text { PAÍS } \\
\text { Seychelles }\end{array}$} & \multicolumn{4}{|c|}{ Modelo 1} & \multicolumn{4}{|c|}{ Modelo 2} \\
\hline & \multirow{2}{*}{$\begin{array}{c}\boldsymbol{O R} \\
0,83\end{array}$} & \multicolumn{2}{|c|}{ IC 95\% } & \multirow{2}{*}{$\begin{array}{c}\boldsymbol{p} \\
0,384\end{array}$} & \multirow{2}{*}{$\begin{array}{c}\boldsymbol{O R} \\
0,86\end{array}$} & \multicolumn{2}{|c|}{ IC 95\% } & \multirow{2}{*}{$\begin{array}{c}\boldsymbol{p} \\
0,488\end{array}$} \\
\hline & & 0,55 & - 1,26 & & & 0,57 & - 1,31 & \\
\hline Uganda & 1,54 & 0,86 & - 1,55 & 0,339 & 1,17 & 0,87 & - 1,58 & 0,299 \\
\hline Argentina & 0,98 & 0,65 & - 1,47 & 0,907 & 1,03 & 0,68 & - 1,57 & 0,874 \\
\hline Cayman Islands & 2,14 & 1,28 & $-3,55$ & 0,002 & 2,05 & 1,22 & $-3,45$ & 0,005 \\
\hline Chile & 1,21 & 1,02 & - 1,42 & 0,024 & 1,28 & 1,08 & - 1,51 & 0,004 \\
\hline Ecuador & 0,85 & 0,62 & - 1,15 & 0,285 & 0,92 & 0,67 & - 1,26 & 0,611 \\
\hline Uruguay & 0,96 & 0,70 & - 1,32 & 0,799 & 1,09 & 0,78 & - 1,52 & 0,607 \\
\hline India & 1,24 & 0,94 & - 1,65 & 0,128 & 1,28 & 0,96 & - 1,69 & 0,089 \\
\hline Indonesia & 1,35 & 0,90 & $-2,03$ & 0,141 & 1,36 & 0,90 & $-2,03$ & 0,138 \\
\hline Myanmar & 0,56 & 0,28 & - 1,14 & 0,108 & 0,58 & 0,28 & - 1,19 & 0,132 \\
\hline Sri lanka & 0,90 & 0,54 & - 1,50 & 0,694 & 0,93 & 0,56 & - 1,56 & 0,782 \\
\hline Thailand & 1,12 & 0,75 & - 1,66 & 0,589 & 1,14 & 0,76 & - 1,69 & 0,528 \\
\hline Djibouti & 1,12 & 0,79 & $-1,60$ & 0,525 & 1,13 & 0,79 & - 1,61 & 0,504 \\
\hline Egypt & 0,97 & 0,80 & - 1,17 & 0,726 & 0,94 & 0,78 & - 1,14 & 0,546 \\
\hline Jordan & 0,85 & 0,62 & - 1,17 & 0,317 & 0,86 & 0,63 & - 1,17 & 0,335 \\
\hline United Arab Emirates & 1,05 & 0,95 & - 1,15 & 0,366 & 1,09 & 0,99 & - 1,21 & 0,082 \\
\hline China & 1,10 & 0,86 & - 1,41 & 0,446 & 1,14 & 0,89 & - 1,46 & 0,306 \\
\hline Philippines & 0,93 & 0,78 & - 1,10 & 0,389 & 0,95 & 0,80 & - 1,14 & 0,595 \\
\hline Test of homogeneity= & & & & 25,56 & & & & 24,50 \\
\hline $\operatorname{Pr}>\operatorname{chi} 2=$ & & & & 0,082 & & & & 0,106 \\
\hline
\end{tabular}

FONTE: Global School-based Student Health Survey (GSHS) - WHO. 


\section{Associação entre sintomas depressivos e obesidade em cada país}

\section{Sexo masculino}

Não encontramos associação entre sintomas depressivos e obesidade para o sexo masculino (Tabela 5, Modelo 2) em nenhum dos 18 países analisados.

\begin{tabular}{|c|c|c|c|c|c|c|c|c|}
\hline \multirow{3}{*}{$\begin{array}{l}\text { PAÍS } \\
\text { Seychelles }\end{array}$} & \multicolumn{4}{|c|}{ Modelo 1} & \multicolumn{4}{|c|}{ Modelo 2} \\
\hline & \multirow{2}{*}{$\begin{array}{c}\text { OR } \\
0,63\end{array}$} & \multicolumn{2}{|c|}{ IC $95 \%$} & \multirow{2}{*}{$\begin{array}{c}\boldsymbol{p} \\
0,245\end{array}$} & \multirow{2}{*}{$\begin{array}{c}\boldsymbol{O R} \\
0,63\end{array}$} & \multicolumn{2}{|c|}{ IC 95\% } & \multirow{2}{*}{$\begin{array}{c}\boldsymbol{p} \\
0,254\end{array}$} \\
\hline & & 0,28 & $-1,39$ & & & 0,29 & $-1,40$ & \\
\hline Uganda & 1,55 & 0,39 & $-6,22$ & 0,534 & 1,48 & 0,36 & $-6,06$ & 0,580 \\
\hline Argentina & 1,28 & 0,66 & $-2,46$ & 0,464 & 1,39 & 0,71 & $-2,70$ & 0,332 \\
\hline Cayman Islands & 1,22 & 0,62 & $-2,37$ & 0,563 & 1,18 & 0,61 & $-2,29$ & 0,613 \\
\hline Chile & 0,84 & 0,62 & $-1,13$ & 0,257 & 0,87 & 0,64 & - 1,17 & 0,354 \\
\hline Ecuador & 1,45 & 0,92 & $-2,27$ & 0,104 & 1,50 & 0,95 & $-2,37$ & 0,077 \\
\hline Uruguay & 1,23 & 0,63 & $-2,39$ & 0,539 & 1,29 & 0,66 & $-2,52$ & 0,447 \\
\hline India & 0,72 & 0,42 & - 1,23 & 0,226 & 0,75 & 0,43 & $-1,28$ & 0,286 \\
\hline Indonesia & 0,49 & 0,19 & $-1,25$ & 0,128 & 0,49 & 0,19 & $-1,26$ & 0,132 \\
\hline Myanmar & 0,00 & . & - & 0,106 & 0,00 & . & - & 0,100 \\
\hline Sri lanka & 1,93 & 0,39 & $-9,64$ & 0,414 & 1,95 & 0,40 & - 9,63 & 0,401 \\
\hline Thailand & 0,85 & 0,50 & - 1,46 & 0,557 & 0,86 & 0,50 & - 1,49 & 0,598 \\
\hline Djibouti & 1,05 & 0,51 & $-2,15$ & 0,903 & 1,10 & 0,54 & $-2,26$ & 0,774 \\
\hline Egypt & 1,04 & 0,77 & - 1,39 & 0,809 & 1,08 & 0,80 & $-1,45$ & 0,606 \\
\hline Jordan & 1,04 & 0,69 & $-1,56$ & 0,864 & 1,04 & 0,69 & $-1,57$ & 0,842 \\
\hline United Arab Emirates & 1,00 & 0,88 & - 1,41 & 0,946 & 1,03 & 0,91 & - 1,17 & 0,639 \\
\hline China & 1,07 & 0,78 & - 1,46 & 0,694 & 1,10 & 0,80 & $-1,51$ & 0,544 \\
\hline Philippines & 0,85 & 0,65 & $-1,10$ & 0,213 & 0,85 & 0,65 & $-1,11$ & 0,226 \\
\hline Test of homogeneity = & & & & 15,94 & & & & 16,86 \\
\hline $\operatorname{Pr}>\operatorname{chi} 2=$ & & & & 0,528 & & & & 0,463 \\
\hline
\end{tabular}

FONTE: Global School-based Student Health Survey (GSHS) - WHO. 


\section{$\underline{\text { Sexo feminino }}$}

Para o sexo feminino, ajustando-se para idade, encontramos associação positiva entre sintomas depressivos e obesidade nas Ilhas Cayman $(O R=2,35$; IC 95\%: 1,16-4,73), no Chile (OR = 1,36; IC 95\%: 1,05-1,76) e nos Emirados Árabes $(O R=1,16 ;$ IC 95\%: 1,01-1,32) (Tabela 6, Modelo 2).

\begin{tabular}{|c|c|c|c|c|c|c|c|c|}
\hline \multirow{3}{*}{$\begin{array}{l}\text { PAÍS } \\
\text { Seychelles }\end{array}$} & \multicolumn{4}{|c|}{ Modelo 1} & \multicolumn{4}{|c|}{ Modelo 2} \\
\hline & \multirow{2}{*}{$\begin{array}{c}\boldsymbol{O R} \\
1,08\end{array}$} & \multicolumn{2}{|c|}{ IC 95\% } & \multirow{2}{*}{$\begin{array}{c}\boldsymbol{p} \\
0,792\end{array}$} & \multirow{2}{*}{$\begin{array}{c}\text { OR } \\
1,12\end{array}$} & \multicolumn{2}{|c|}{ IC $95 \%$} & \multirow{2}{*}{$\begin{array}{c}p \\
0,702\end{array}$} \\
\hline & & 0,62 & - 1,89 & & & 0,63 & $-1,97$ & \\
\hline Uganda & 1,17 & 0,47 & $-2,89$ & 0,738 & 1,23 & 0,50 & $-3,04$ & 0,653 \\
\hline Argentina & 1,30 & 0,52 & $-3,24$ & 0,568 & 1,45 & 0,57 & $-3,67$ & 0,429 \\
\hline Cayman Islands & 2,45 & 1,21 & $-4,96$ & 0,009 & 2,35 & 1,16 & $-4,73$ & 0,014 \\
\hline Chile & 1,26 & 0,98 & $-1,62$ & 0,076 & 1,36 & 1,05 & - 1,76 & 0,019 \\
\hline Ecuador & 0,70 & 0,40 & - 1,22 & 0,208 & 0,72 & 0,42 & $-1,25$ & 0,246 \\
\hline Uruguay & 1,02 & 0,50 & $-2,07$ & 0,958 & 1,24 & 0,60 & $-2,55$ & 0,557 \\
\hline India & 1,51 & 0,76 & $-3,03$ & 0,238 & 1,53 & 0,76 & $-3,08$ & 0,227 \\
\hline Indonesia & 0,99 & 0,40 & - 2,47 & 0,980 & 1,05 & 0,42 & $-2,63$ & 0,925 \\
\hline Myanmar & 0,00 & r & - & 0,207 & 0,00 & . & - & 0,228 \\
\hline Sri lanka & 0,55 & 0,16 & $-1,98$ & 0,355 & 0,56 & 0,16 & $-2,01$ & 0,367 \\
\hline Thailand & 0,90 & 0,45 & - 1,80 & 0,772 & 0,93 & 0,47 & $-1,84$ & 0,827 \\
\hline Djibouti & 1,15 & 0,63 & $-2,10$ & 0,650 & 1,19 & 0,64 & $-2,18$ & 0,583 \\
\hline Egypt & 1,10 & 0,80 & - 1,52 & 0,554 & 1,07 & 0,78 & $-1,48$ & 0,676 \\
\hline Jordan & 0,96 & 0,52 & - 1,78 & 0,896 & 0,97 & 0,52 & $-1,80$ & 0,916 \\
\hline United Arab Emirates & 1,13 & 0,99 & - 1,29 & 0,074 & 1,16 & 1,01 & $-1,32$ & 0,033 \\
\hline China & 1,05 & 0,68 & $-1,66$ & 0,804 & 1,09 & 0,69 & - 1,71 & 0,712 \\
\hline Philippines & 1,10 & 0,83 & - 1,46 & 0,489 & 1,11 & 0,83 & - 1,47 & 0,481 \\
\hline Test of homogeneity = & & & & 13,31 & & & & 13,46 \\
\hline $\operatorname{Pr}>\operatorname{chi} 2=$ & & & & 0,715 & & & & 0,705 \\
\hline
\end{tabular}

FONTE: Global School-based Student Health Survey (GSHS) - WHO. 


\section{Teste de Homogeneidade (TH)}

As associações tanto entre sintomas depressivos e sobrepeso (Tabelas 3

e 4), quanto entre sintomas depressivos e obesidade (Tabelas 5 e 6), para ambos os sexos, mostraram-se homogêneas entre os países ( $p>0,05$ no Test of Homogeneity - TH).

\section{Associação entre sintomas depressivos e sobrepeso e obesidade na amostra total}

A partir dos resultados do $T H$ nós calculamos o ORs para estimativa da associação entre sintomas depressivos e sobrepeso, e sintomas depressivos e obesidade para a amostra total, composta por todos os adolescentes de todos os 18 países estudados (Tabela 7).

\begin{tabular}{|c|c|c|c|c|c|c|}
\hline & \multicolumn{3}{|c|}{ Masculino } & \multicolumn{3}{|c|}{ Feminino } \\
\hline & OR & IC 95\% & $p$ & OR & IC 95\% & $p$ \\
\hline \multicolumn{7}{|l|}{ Sobrepeso } \\
\hline Modelo 1 & 0,94 & $0,89-0,99$ & 0,016 & 1,15 & $1,09-1,22$ & $<0,01$ \\
\hline Modelo 2 & 1,01 & $0,96-1,07$ & 0,702 & 1,24 & $1,18-1,31$ & $<0,01$ \\
\hline \multicolumn{7}{|l|}{ Obesidade } \\
\hline Modelo 1 & 0,99 & $0,91-1,07$ & 0,753 & 1,25 & $1,15-1,36$ & $<0,01$ \\
\hline Modelo 2 & 1,04 & $0,96-1,13$ & 0,319 & 1,33 & $1,22-1,46$ & $<0,01$ \\
\hline
\end{tabular}

FONTE: Global School-based Student Health Survey (GSHS) - WHO.

Encontramos associação positiva de sintomas depressivos com sobrepeso e de sintomas depressivos com obesidade em ambos os modelos de análise (Tabela 7, Modelos 1 e 2), mas apenas para o sexo feminino, e não para o sexo masculino. 


\section{DISCUSSÃo}

Como esperávamos, houve grande variabilidade dentre os países e entre os sexos nas prevalências de sintomas depressivos, sobrepeso e obesidade.

Encontramos associações entre sintomas depressivos e sobrepeso e sintomas depressivos e obesidade para adolescentes do sexo feminino quando analisamos a amostra total, composta por todos os adolescentes de todos os 18 países.

Contudo, não encontramos a associação esperada ente sintomas depressivos e excesso de peso nos adolescentes quando estimamos separadamente para cada país. Nós supúnhamos que dentre os diversos contextos socioculturais, níveis de desenvolvimento e diferentes prevalências de sintomas depressivos, sobrepeso e obesidade, encontraríamos associações de diferentes direções e intensidades. Entretanto, encontramos associação na minoria dos países, o que nos impediu de podermos comparar essas associações de acordo com os diferentes contextos de cada país.

A associação encontrada para a amostra total, composta por países tão diversos culturalmente, pode indicar que sintomas depressivos pode estar associado com sobrepeso e obesidade em adolescentes do sexo feminino independente do contexto sociocultural de onde elas vivem. Porém, consideramos necessário ressaltar que nosso estudo não avaliou os contextos socioculturais dos países estudados. 


\section{Comparação com outros estudos}

Scott e col. (2008), assim como nós, estudaram inquéritos de diversos países e encontraram ORs significantes para a associação entre obesidade e desordens depressivas em adultos quando analisada a amostra com todos os países. Contudo, os ORs calculados para cada país foram significantes apenas na minoria deles também (50).

Em relação ao sexo como moderador da associação, nossos resultados concordam com os resultados publicados em outros estudos transversais (42, $50,85,86)$. Nestes estudos o sexo também foi moderador da relação depressãoobesidade, com associação entre sintomas depressivos e obesidade presente nas mulheres, mas não nos homens.

Quando examinada a associação bidirecional entre depressão e obesidade, estudos prospectivos encontraram resultados mistos. Alguns encontraram influência de obesidade em subsequente depressão, mas não de depressão em subsequente obesidade $(57,88)$. Uma meta-análise de estudos longitudinais encontrou associação bidirecional (56), e outros estudos prospectivos encontraram que depressão durante infância e adolescência esteve associada com risco aumentado de obesidade em maior idade $(39,40,58-60,89)$.

Como mostramos até agora, existem diversos estudos transversais e longitudinais investigando a associação entre depressão e obesidade. Embora a maior parte deles tenha sido conduzida em população norte americana, há outros que foram conduzidos em outros países e regiões como França, Holanda, Nova Zelândia (NZ), Suíça e Reino Unido, por exemplo. Porém, ainda existe uma grande concentração de pesquisas sobre esse assunto nos países e regiões desenvolvidos, com poucos estudos envolvendo países em desenvolvimento. 
Nossos resultados sugerem que sintomas depressivos estão associados com sobrepeso e obesidade em adolescentes do sexo feminino, de países que não os EUA, NZ ou países europeus.

\section{Relação entre depressão e obesidade}

Consideramos importante abordar alguns potenciais mecanismos pelos quais depressão e sintomas depressivos poderiam promover sobrepeso e obesidade.

\section{Comer emocional}

Estudos mostram que o comer emocional (Emotional Eating (EmE)) esta positivamente relacionado com sintomas depressivos (61-64). Alguns pesquisadores ainda sugerem que o comer emocional poderia ser um dos fatores que explicariam a associação entre sintomas depressivos e a adiposidade (61, 62), assim como a associação com consumo de alimentos doces e de maior densidade calórica (63). Outro estudo relatou que o comer emocional mostrouse emergir durante a adolescência em relação direta com sintomas depressivos (90).

Hiperfagia

Outro potencial sintoma em indivíduos com depressão atípica é a hiperfagia, já que comer em excesso (overeating) é uma das características que podem aparecem em indivíduos com esse transtorno (65-67). 


\section{Estilo de vida}

Aumento da ingestão de alimentos e diminuição da prática de atividade física são considerados como sintomas de depressão pelo Manual Estatístico de Diagnóstico de Desordens Mentais, quarta edição (Diagnostic and Statistical Manual of Mental Disorders (DSM-IV)).

Sendo assim, a adoção de um estilo de vida não saudável, com prática insuficiente de atividade física e preferências alimentares não saudáveis, pode ocorrer em conjunto com sintomas depressivos, possivelmente levando ao ganho de peso $(42,43,68,69)$.

\section{Qualidade do sono}

Um padrão de sono ruim é conhecido como fator de riso para desenvolvimento de obesidade (76). É bastante comum que os indivíduos afetados pela depressão sofram com sono de má qualidade (70). Portanto, outro potencial caminho pelo qual a depressão poderia promover a obesidade seria através da qualidade do sono.

\section{Distúrbios neuroendócrinos}

O fato de que depressão pode causar um aumento de peso com o passar do tempo pode também ser decorrência de distúrbios neuroendócrinos. A relação da depressão com aumento de peso pode ser explicada por inflamação ou desregulação de leptina, cortisol ou da HPA axis (71-75). 
Medicações Psicotrópicas

Finalmente, algumas medicações psicotrópicas, como os antidepressivos, por exemplo, estão associadas com uma indesejada mudança de peso $(37,77$ 79). Entretanto, os resultados de estudos que usaram diagnóstico clínico para medir depressão, onde os indivíduos usualmente são tratados com antidepressivos, não foram diferentes dos resultados dos estudos que usaram sintomas depressivos.

\section{Limitações}

A primeira limitação do nosso estudo é seu corte transversal, que nos impossibilita inferir causalidade na relação de sintomas depressivos com excesso de peso. Ou seja, através de nossos resultados não podemos dizer se sintomas depressivos levaram os adolescentes a ganharem peso, ou se o excesso de peso fez com que eles desenvolvessem sintomas depressivos.

\section{$\underline{\text { IMC auto-referido }}$}

Como descrevemos anteriormente nos métodos do nosso trabalho, estimamos os valores de IMC a partir de valores de altura e peso auto-referidos. Algumas evidências sugerem que dados de peso e altura auto-referidos subestimam a prevalência de sobrepeso e obesidade em adolescentes (91-93). Baseando-se nessa premissa, as associações entre sintomas depressivos e excesso de peso encontradas em nosso estudo poderiam estar atenuadas em relação às suas verdadeiras magnitudes em adolescentes. 


\section{Sintomas depressivos}

Utilizamos como indicativo de sintomas depressivos uma questão do questionário da GSHS que é auto-aplicado, ou seja, os alunos respondem sozinhos a todas as questões e não há entrevista. Isso é uma limitação pois essa questão não nos permitiu realizar um diagnóstico de depressão, caracterizar o subtipo ou intensidade da depressão, e não leva em conta a frequente ocorrência de comorbidade com outros transtornos mentais ou psicopatologias passadas.

\section{Variáveis de controle}

Devido à limitações das bases de dados que utilizamos no trabalho, nós não pudemos controlar as análises para contextos sociais como etnia e status socioeconômico (SES), assim como para uso de medicações psicotrópicas. Contudo, a maioria dos estudos prévios que testaram a influência de etnia e $S E S$ na associação entre depressão e obesidade não encontrou evidência $(57,58,86)$. Ainda, nossos resultados pouco possivelmente poderiam ser explicados pelo uso de medicações psicotrópicas, pois trabalhamos com adolescentes e espera-se que o uso dessas medicações em adolescentes seja baixo (94).

\section{Contribuições do presente estudo}

A depressão, independente do nível de severidade que esteja, se deixada sem tratamento pode impactar negativamente na saúde e no funcionamento dos indivíduos afetados (4). Como já mostramos, estudos recentes confirmaram evidências de que pessoas com sintomas depressivos, depressão com características atípicas ou transtorno depressivo maior, têm risco elevado de ganho de peso. 
Apesar de muitos estudos já terem examinado a relação entre depressão e obesidade, muito poucos envolveram adolescentes de diversos contextos socioculturais. A abrangente amostra da GSHS nos ofereceu uma ferramenta única para estudar a associação entre sintomas depressivos e excesso de peso entre adolescentes de diversos países que raramente são envolvidos em estudos mundialmente.

Embora nosso estudo, assim como diversos outros, sugere que sintomas depressivos estão associados com excesso de peso, existem atualmente poucos modelos de tratamento dessas duas condições simultaneamente.

Mesmo que uma direção causal não possa ser determinada pelos nossos resultados, nós consideramos que sintomas depressivos deveriam ser investigados em pessoas em tratamento de sobrepeso e obesidade, assim como indivíduos com depressão ou sintomas depressivos deveriam ser considerados em risco de ganho de peso. 


\section{CONCLUSÕES}

Nosso resultados confirmam a associação entre sintomas depressivos e excesso de peso em adolescentes do sexo feminino, e sugerem que contextos socioculturais de diferentes países poderiam não exercer influência sobre essa associação.

O devido reconhecimento da importância dessa associação pode ajudar na formulação de intervenções com objetivo de melhorar a saúde mental e o combate à epidemia mundial de sobrepeso e obesidade.

Estudos futuros que elucidem mais claramente os fatores causais e mediadores podem auxiliar na identificação e criação de programas e políticas de medidas preventivas e de tratamentos efetivos para a depressão e o excesso de peso. 


\section{ANEXO}

ANEXO A - Questionário principal da GSHS
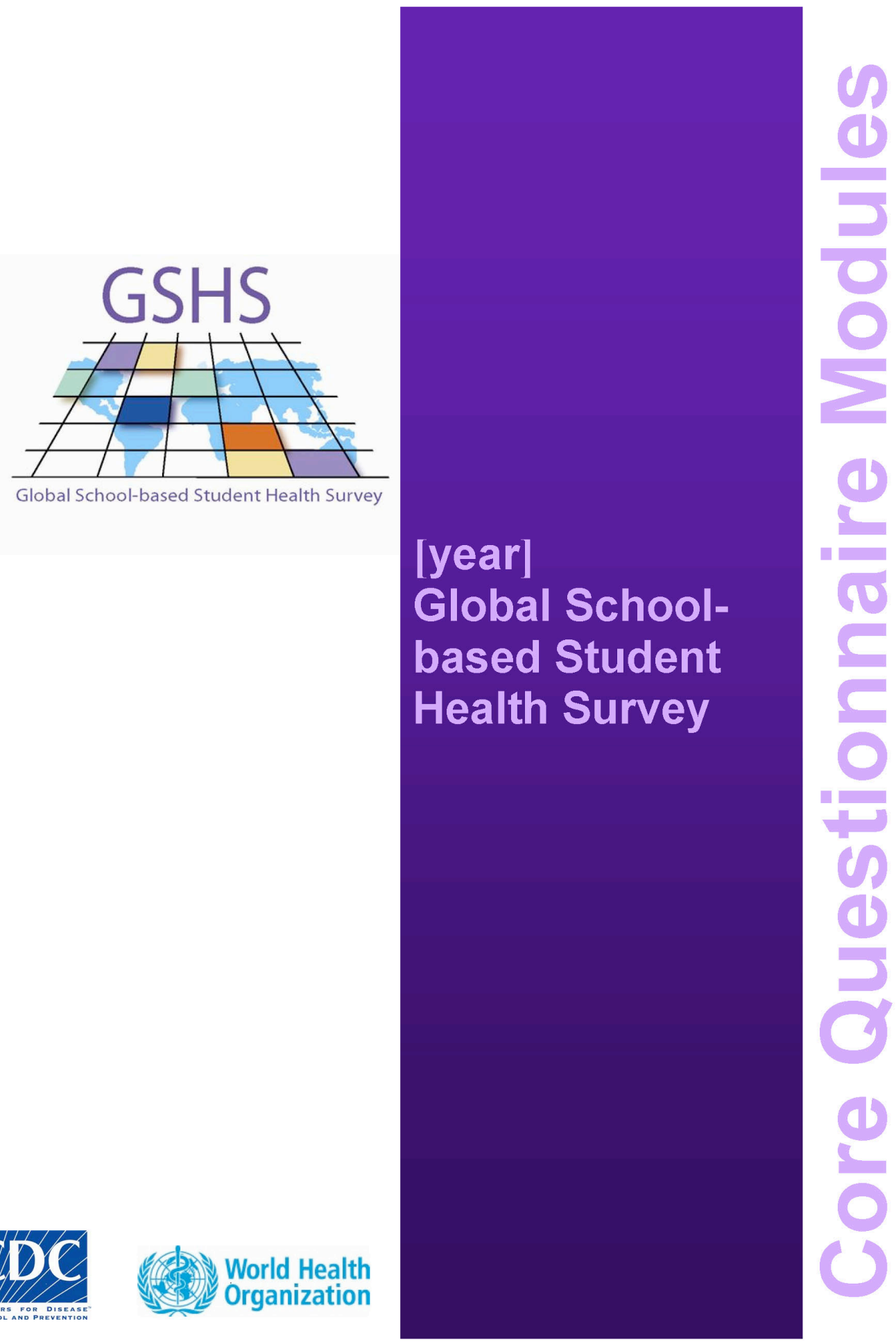


\section{Introduction}

Overview This survey is about your health and the things you do that may affect your health. The information you give will be used to develop better health programs for young people like yourself

Who is doing Students like you all over your country are doing this survey. Students in the survey many other countries around the world also are doing this survey.

Voluntary Completing the survey is voluntary. Your grade or mark in this class will not participation be affected whether or not you answer the questions. If you do not want to answer a question, just leave it blank.

Private DO NOT write your name on this survey or the answer sheet. The answers participation you give will be kept private. No one will know how you answer.

Instructions Follow the instructions below to complete the answer sheet.

- DO NOT write your name on this survey or the answer sheet.

- Use only the GSHS pencil you are given.

- Read every question.

- Answer the questions based on what you really know or do. There are no right or wrong answers.

- Fill in the circles on your answer sheet that match your answer.

- When you are finished, do what the person who is giving you the survey says to do.

Example Here is an example of how to fill in the circles:

Fill in the circles like this

Not like this or

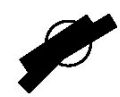

Survey

1. Do fish live in water?
A. Yes

B. No

Answer sheet

1.

(B) (C) (D) () (

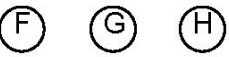

Thank you very much for your help. 


\section{Respondent Demographics Module}

1. How old are you?

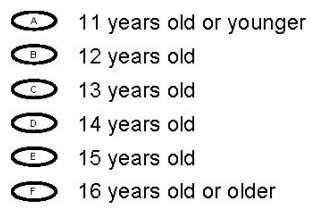

2. What is your sex?

\section{(B) Male \\ E Female}

3. In what grade/class/standard are you? COUNTRY SPECIFIC RESPONSE OPTIONS

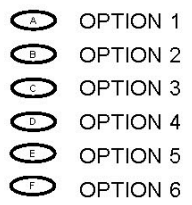




\section{Alcohol and Other Drug Use Module}

The next 5 questions ask about drinking alcohol. This includes drinking COUNTRY SPECIFIC EXAMPLES. Drinking alcohol does not include drinking a few sips of wine [or COUNTRY SPECIFIC EXAMPLES] for religious purposes.

1. During the past 30 days, on how many days did you have at least one drink containing alcohol?

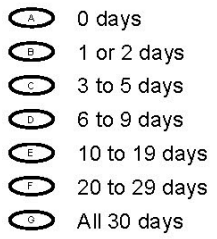

2. During the past 30 days, on the days you drank alcohol, how many drinks did you usually drink per day?

I did not drink alcohol during the past 30 days
Less than one drink
1 drink
2 drinks
B drinks
B drinks
5 or more drinks

3. During the past 30 days, how did you usually get the alcohol you drank? SELECT ONLY ONE RESPONSE.

$\rightarrow$ I did not drink alcohol during the past 30 days B I bought it in a store, shop, or from a street vendor

I gave someone else money to buy it for me I got it from friends E I got it from home

P I stole it

$\$$ I made it myself (DELETE IF NOT

APPROPRIATE in SPECIFIC COUNTRY)

BI got it some other way

4 During your life, how many times did you drink so much alcohol that you were really drunk?

(A) 0 times

D 1 or 2 times

(D) 3 to times

D 10 or more times

5 During your life, how many times have you ever had a hang-over, felt sick, got into trouble with your family or friends, missed school, or got into fights, as a result of drinking alcohol?

(B) 0 times

(B) 1 or 2 times

C) 3 to 9 times

D 10 or more times

The next question asks about drugs.

6. During your life, how many times have you used drugs such as COUNTRY SPECIFIC EXAMPLES?

(A) 0 times

(E) 1 or 2 times

C) 3 to 9 times

(D) 10 or more times 


\section{Dietary Behaviours Module}

The next 3 questions ask about your height, weight, and going hungry.

1. How tall are you without your shoes on? ON THE ANSWER SHEET, WRITE YOUR HEIGHT IN THE SHADED BOXES AT THE TOP OF THE GRID.

THEN FILL IN THE OVAL BELOW EACH NUMBER.

\section{Example}
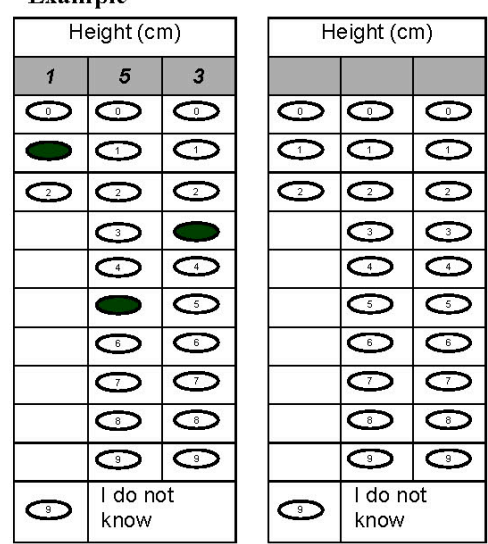

2. How much do you weigh without your shoes on? ON THE ANSWER SHEET, WRITE YOUR WEIGHT IN THE SHADED BOXES AT THE TOP OF THE GRID. THEN FILL IN THE OVAL BELOW EACH NUMBER
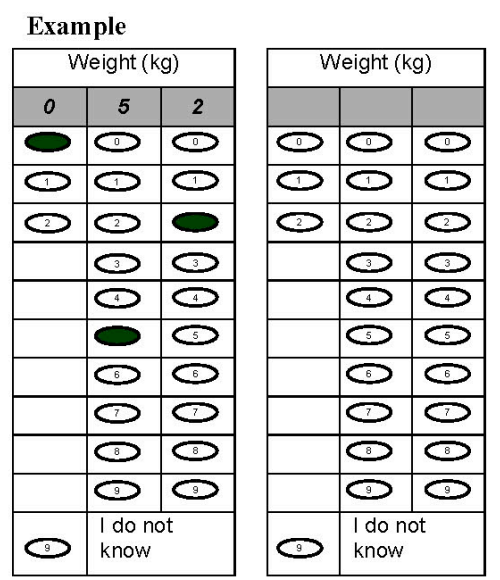

3. During the past 30 days, how often did you go hungry because there was not enough food in your home?

$\rightarrow$ Never

$\triangle$ Rarely

Cometimes

D Most of the time

E Always

The next 2 questions ask about foods you might eat.

4. During the past 30 days, how many times per day did you us ually eat fruit, such as COUNTRY SPECIFIC EXAMPLES?

A I did not eat fruit during the past 30 days

(B) Less than one time per day

C 1 time per day

D 2 times per day

E 3 times per day

F 4 times per day

(S) 5 or more times per day

5. During the past 30 days, how many times per day did you usually eat vegetables, such as COUNTRY

SPECIFIC EXAMPLES?

A I did not eat vegetables during the past 30 days

Dess than one time per day

C 1 time per day

D 2 times per day

E 3 times per day

F 4 times per day

C) 5 or more times per day

Part 4: GSHS Questionnaire 


\section{Hygiene Module}

The next 4 questions ask about cleaning your teeth and washing your hands

1. During the past 30 days, how many times per day did you usually clean or brush your teeth?

A I did not clean or brush my teeth during the past 30 days

$\otimes$ Less than 1 time per day

(S) 1 time per day

$\infty 2$ times per day

E 3 times per day

B 4 or more times per day

2. During the past 30 days, how often did you wash your hands before eating?
$\Leftrightarrow$ Never
Barely
$C$ Sometimes
$D$ Most of the time
E Always

3. During the past 30 days, how often did you wash your hands after using the toilet or latrine?
A Never
B) Rarely
$C$ Sometimes
Most of the time
E Always

4. During the past 30 days, how often did you use soap when washing your hands?
$\Phi$ Never
D Rarely
CS Sometimes
$D$ Most of the time
$B$ Always 


\section{Mental Health Module}

The next 6 questions ask about your feelings and friendships

1. During the past 12 months, how often have you felt lonely?

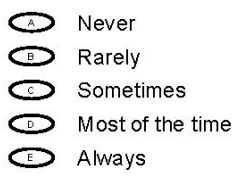

2. During the past 12 months how often have you been so worried about something that you could not sleep at night?

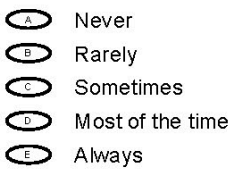

3. During the past 12 months, did you ever feel so sad or hopeless almost every day for two weeks or more in a row that you stopped doing your usual activities?

$\Leftrightarrow$ Yes

Do

4. During the past 12 months, did you ever seriously consider attempting suicide?

\section{$\triangle$ Yes}

$\rightarrow$ No

5. During the past 12 months, did you make a plan about how you would attempt suicide?

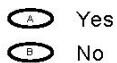

6. How many close friends do you have?

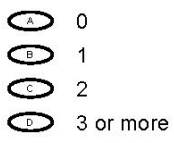

Part 4: GSHS Questionnaire 


\section{Physical Activity Module}

The next 2 questions ask about physical activity. Physical activity is any activity that increases your heart rate and makes you get out of breath some of the time. Physical activity can be done in sports, playing with friends, or walking to school. Som examples of physical activity are running, fast walking, biking, dancing, football, and COUNTRY SPECIFIC EXAMPLES.

ADD UP ALL THE TIME YOU SPEND IN PHYSICAL ACTIVITY EACH DAY. DO NOT INCLUDE YOUR PHYSICAL EDUCATION OR GYM CLASS.

1. During the past 7 days, on how many days were you physically active for a total of at least 60 minutes per dav?

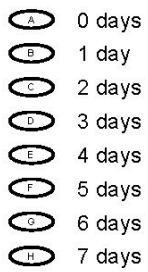

2. During a typical or usual week, on how many days are you physically active for a total of at least 60 minutes per day?

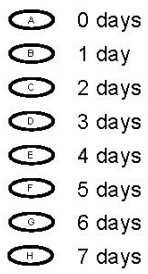

The next question asks about the time you spend mostly sitting when you are not in school or doing homework.

3. How much time do you spend during a typical or usual day sitting and watching television, playing computer games, talking with friends, or doing other sitting activities, such as COUNTRY SPECIFIC EXAMPLES?

A Less than 1 hour per day

(B) 1 to 2 hours per day

(C) 3 to 4 hours per day

5 to 6 hours per day

E 7 to 8 hours per day

F) More than 8 hours per day

The next 2 questions ask about going to and coming home from school.

4. During the past 7 days, on how many days did you walk or ride a bicycle to and from school?

(A) 0 days

D 1 day

(D) 2 days

D 3 days

E 4 days

5 days

(3) 6 days

स 7 days

5. During the past 7 days, how long did it usually take for you to get to and from school each day? ADD UP THE TIME YOU SPEND GOING TO AND COMING HOME FROM SCHOOL.

A Less than 10 minutes per day

B 10 to 19 minutes per day

C 20 to 29 minutes per day

D 30 to 39 minutes per day

E 40 to 49 minutes per day

F 50 to 59 minutes per day

(5) 60 or more minutes per day 


\section{Protective Factors Module}

The next 5 questions as $k$ about your experiences at school and at home.

1. During the past 30 days, on how many days did you miss classes or school without permission?

A 0 days

ब1 1 or 2 days

3 to 5 days

D 6 to 9 days

E 10 or more days

2. During the past 30 days, how often were most of the students in your school kind and helpful?
A) Never
B Rarely
Sometimes
Most of the time
E Always

3. During the past 30 days, how often did your parents or guardians check to see if your homework was done?

B Never

(a) Rarely

(C) Sometimes

Dost of the time

E Always

4. During the past 30 days, how often did your parents or quardians understand your problems and worries?

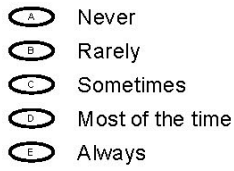

During the past 30 days, how often did your parents or guardians really know what you were doing with your free time?
A Never
Direly
$D$ Sometimes
Dost of the time
E Always 


\section{Sexual Behaviours that Contribute to HIV Infection, Other STI, and Unintended Pregnancy Module}

The next 5 questions ask about sexual intercourse.

1. Have you ever had sexual intercourse?

$\triangle$ Yes

$\rightarrow$ No

2. How old were you when you had sexual intercourse for the first time?

B I have never had sexual intercourse

(D) 11 years old or younger

C) 12 years old

D 13 years old

E 14 years old

$\Leftrightarrow 15$ years old

(G) 16 years old or older

3. During your life, with how many people have you had sexual intercourse?

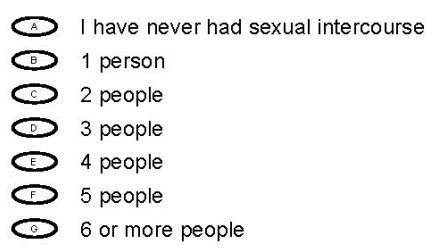

5. During the past 12 months, have you had sexual intercourse?

$\rightarrow$ Yes

$\rightarrow$ No
5. The last time you had sexual intercourse, did you or your partner use a condom or [COUNTRY SPECIFIC SLANG TERM FOR CONDOM]?

$\triangle$ I have never had sexual intercourse

Q Yes

C No 


\section{Tobacco Use Module}

The next 6 questions ask about cigarette and other tobacco use.

1. How old were you when you first tried a cigarette?

A I have never smoked cigarettes

(D) 7 years old or younger

C) 8 or 9 years old

D 10 or 11 years old

E 12 or 13 years old

E 14 or 15 years old

(5) 16 years old or older

2. During the past 30 days, on how many days did you smoke cigarettes?
A 0 days
(9) 1 or 2 days
(S) 3 to 5 days
D 6 to 9 days
E 10 to 19 days
B 20 to 29 days
All 30 days

3. During the past 30 days, on how many days did you use any other form of to bacco, such as COUNTRY SPECIFIC EXAMPLES?

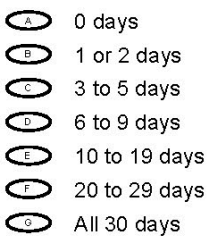

4. During the past 12 months, have you ever tried to stop smoking cigarettes?

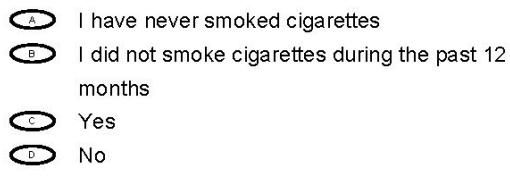

5. During the past 7 days, on how many days have people smoked in your presence?

A 0 days

D 1 or 2 days

C) 3 or 4 days

D 5 or 6 days

E All 7 days

6. Which of your parents or guardians use any form of to bacco?

$B$ Neither

$\Xi$ My father or male guardian

C My mother or female guardian

Both

E I do not know 


\section{Violence and Unintentional Injury Module}

The next question asks about physical attacks. A physical attack occurs when one or more people hit or strike someone, or when one or more people hurt another person with a weapon (such as a stick, knife or gun). It is not a physical attack when two student of about the same strength or power choose to fight each other.

1. During the past 12 months how many times were you physically attacked?
(A) 0 times
D 1 time
C 2 or 3 times
4 or 5 times
(5) or 7 times
8 or 9 times
(G) 10 or 11 times
(H) 12 or more times

The next question asks about physical fights. A physical fight occurs when two or more students of about the same strength or power choose to fight each other.

2. During the past 12 months, how many times were you in a physical fight?

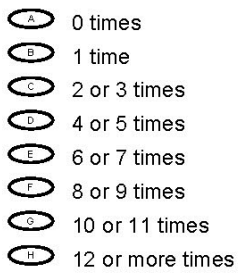

The next 4 questions ask about the most serious injury that happened to you during the past 12 months. An injury is serious when it makes you miss at least one full day of usual activities (such as school, sports, or a job) or requires treatment by a doctor or nurse.
3. During the past 12 months, what were you doing when the most serious injury happened to you?

A I was not seriously injured during the past 12 months

D Playing or training for a sport

C) Walking or running, but not as part of playing or training for a sport

$D$ Riding a bicycle, scooter, or (OTHER COUNTRY SPECIFIC NON-MOTORIZED FORM OF TRANSPORTATION)

E Riding or driving in a car or other motor vehicle

(B) Doing any paid or unpaid work, including housework, yard work, or cooking

(G) Nothing

H) Something else

4. During the past 12 months, what was the major cause of the most serious injury that happened to you?

(A) I was not seriously injured during the past 12 months

(B) I was in a motor vehicle accident or hit by a motor vehicle

$C \mid$ fell

$D$ Something fell on me or hit me

(E) I was fighting with someone

F I was attacked, assaulted, or abused by someone

I I was in a fire or too near a flame or something hot

(H) Something else caused my injury

5. During the past 12 months, how did the most serious injury happen to you?

A I was not seriously injured during the past 12 months

(B) I hurt myself by accident

Someone else hurt me by accident

D I hurt myself on purpose

E Someone else hurt me on purpose 
Violence and Unintentional Injury Module, continued

6. During the past 12 months, what was the most serious injury that happened to you?

A I was not seriously injured during the past 12 months

ब I had a broken bone or a dislocated joint

I I had a cut, puncture, or stab wound

$\Phi$ I had a concussion or other head or neck injury, was knocked out, or could not breathe

E I had a gunshot wound

F I had a bad burn

S I lost all or part of a foot, leg, hand, or arm

B Something else happened to me

The next question asks about bullying. Bullying

occurs when a student or group of students say or do

bad and unpleasant things to another student. It is

also bullying when a student is teased a lot in an

unpleasant way or when a student is left out of things

on purpose. It is not bullying when two students of

about the same strength or power argue or fight or

when teasing is done in a friendly and fun way.

7. During the past 30 days, how were you bullied most often?

A I was not bullied during the past 30 days

(]) I was hit, kicked, pushed, shoved around, or locked indoors

I I was made fun of because of my race or colour

$D$ I was made fun of because of my religion

$E$ I was made fun of with sexual jokes, comments, or gestures

$\notin$ I was left out of activities on purpose or completely ignored

$\rightarrow$ I was made fun of because of how my body or face looks

H I was bullied in some other way 


\section{REFERÊNCIAS BIBLIOGRÁFICAS}

1. World Health Organization (WHO). Media Centre. Fact Sheets: Depression, $\quad \mathrm{n}^{0}$ : $\quad 369 ; \quad 2015 . \quad$ Disponível em: <http://www.who.int/mediacentre/factsheets/fs369/en/> Acesso em: 15 fevereiro 2016.

2. Murray CJL, Vos T, Lozano R, AlMazroa MA, Memish ZA. Disabilityadjusted life years (DALYs) for 291 diseases and injuries in 21 regions, 19902010: a systematic analysis for the Global Burden of Disease Study 2010 (vol 380, pg 2197, 2012). Lancet. 2013;381(9867):628.

3. Vos T, Flaxman AD, Naghavi M. Years lived with disability (YLDs) for 1160 sequelae of 289 diseases and injuries 1990-2010: a systematic analysis for the Global Burden of Disease Study 2010 (vol 380, pg 2163, 2012). Lancet. 2014;384(9943):582-.

4. Prince M, Patel V, Shekhar S, Maj M, Maselko J, Phillips MR, et al. Global mental health 1 - No health without mental health. Lancet. 2007;370(9590):85977.

5. Atlantis E, Goldney RD, Eckert KA, Taylor AW, Phillips P. Trends in healthrelated quality of life and health service use associated with comorbid diabetes and major depression in South Australia, 1998-2008. Social Psychiatry and Psychiatric Epidemiology. 2012;47(6):871-7.

6. Cuijpers P, Vogelzangs N, Twisk J, Kleiboer A, Li J, Penninx BW. Comprehensive Meta-Analysis of Excess Mortality in Depression in the General Community Versus Patients With Specific Illnesses. American Journal of Psychiatry. 2014;171(4):453-62.

7. World Health Organization (WHO). Media Centre. Fact Sheets: Adolescents: health risks and solutions, $\mathrm{n}^{\circ}$ : 345 ; 2016. Disponível em: <http://www.who.int/mediacentre/factsheets/fs345/en/> Acesso em: 20 maio 2016.

8. Instituto Brasiliero de Geografia e Estatística - IBGE. Pesquisa Nacional por Amostra de Domicílio 2008: Um panorama de saúde no Brasil. Publicado em 31 de março de 2010. Disponível em: <http://www.ibge.gov.br/home/presidencia/noticias/noticia_visualiza.php?id_n oticia $=1580 \&>$. Acesso em: janeiro de 2013.

9. Munhoz TN, Nunes BP, Wehrmeister FC, Santos IS, Matijasevich A. A nationwide population-based study of depression in Brazil. Journal of Affective Disorders. 2016;192:226-33. 
10. Adams KF, Schatzkin A, Harris TB, Kipnis V, Mouw T, Ballard-Barbash R, et al. Overweight, obesity, and mortality in a large prospective cohort of persons 50 to 71 years old. New England Journal of Medicine. 2006;355(8):763-78.

11. World Health Organization (WHO). Media Centre. Fact Sheets: Obesity and overweight, $\mathrm{n}^{\circ} 311$, January 2015. Disponível em: <http://www.who.int/mediacentre/factsheets/fs311/en/>. Acesso em: 15 fev. 2016.

12. Ogden CL, Carroll M. Prevalence of Obesity Among Children and Adolescents: United States, Trends 1963-1965 Trough 2007-2008. Division of Health and Nutrition Examination Surveys. 2010.

13. Ogden, Carroll MD, Kit BK, Flegal KM. Prevalence of Obesity in the United States, 2009-2010. U.S. DEPARTMENT OF HEALTH AND HUMAN SERVICES. Centers for Disease Control and Prevention. Disponível em: <http://www.cdc.gov/nchs/data/databriefs/db82.pdf>. Acesso em: janeiro 2013.

14. Ong KK, Loos RJF. Rapid infancy weight gain and subsequent obesity: Systematic reviews and hopeful suggestions. Acta Paediatrica. 2006;95(8):904-8.

15. Monteiro POA, Victora CG. Rapid growth in infancy and childhood and obesity in later life - a systematic review. Obesity Reviews. 2005;6(2):143-54.

16. Freedman DS, Khan LK, Dietz WH, Srinivasan SR, Berenson GS. Relationship of childhood obesity to coronary heart disease risk factors in adulthood: The Bogalusa Heart Study. Pediatrics. 2001;108(3):712-8.

17. Freedman DS, Dietz WH, Srinivasan SR, Berenson GS. The relation of overweight to cardiovascular risk factors among children and adolescents: The Bogalusa heart study. Pediatrics. 1999;103(6):1175-82.

18. Johnson WD, Kroon JJM, Greenway FL, Bouchard C, Ryan D, Katzmarzyk PT. Prevalence of Risk Factors for Metabolic Syndrome in Adolescents National Health and Nutrition Examination Survey (NHANES), 2001-2006. Archives of Pediatrics \& Adolescent Medicine. 2009;163(4):371-7.

19. Ludwig DS, Ebbeling CB. Type 2 diabetes mellitus in children - Primary care and public health considerations. Jama-Journal of the American Medical Association. 2001;286(12):1427-30.

20. Tounian P, Aggoun Y, Dubern B, Varille V, Guy-Grand B, Sidi D, et al. Presence of increased stiffness of the common carotid artery and endothelial dysfunction in severely obese children: a prospective study. Lancet. 2001;358(9291):1400-4. 
21. Guo SS, Chumlea WC. Tracking of body mass index in children in relation to overweight in adulthood. American Journal of Clinical Nutrition. 1999;70(1):145S-8S.

22. Serdula MK, Ivery D, Coates RJ, Freedman DS, Williamson DF, Byers T. DO OBESE CHILDREN BECOME OBESE ADULTS - A REVIEW OF THE LITERATURE. Preventive Medicine. 1993;22(2):167-77.

23. Whitaker RC, Wright JA, Pepe MS, Seidel KD, Dietz WH. Predicting obesity in young adulthood from childhood and parental obesity. New England Journal of Medicine. 1997;337(13):869-73.

24. Felitti VJ. CHILDHOOD SEXUAL ABUSE, DEPRESSION, AND FAMILY DYSFUNCTION IN ADULT OBESE PATIENTS - A CASE-CONTROL STUDY. Southern Medical Journal. 1993;86(7):732-6.

25. Instituto Brasileiro de Geografia e Estatísitca - IBGE. Pesquisa de Orçamentos Familiares 2008-2009: antropometria e estado nutricional de crianças, adolescentes e adultos no Brasil. Rio de Jnaeiro, 2010. 130p.

26. Sichieri R, do Nascimento S, Coutinho W. The burden of hospitalization due to overweight and obesity in Brazil. Cadernos De Saude Publica. 2007;23(7):1721-7.

27. Porto EBS, Morais TW, Raso V. Avaliação do nível de conhecimento multidiscilpinar dos futuros profissionais na propedêutica da obesidade. RevBrasNutr Emagrecimento 2007;1(2):67-71.

28. Mathers CD, Loncar D. Projections of global mortality and burden of disease from 2002 to 2030. Plos Medicine. 2006;3(11).

29. Murray CJL, Lopez AD. Alternative projections of mortality and disability by cause 1990-2020: Global burden of disease study. Lancet. 1997;349(9064):1498-504.

30. Allison DB, Zannolli R, Narayan KMV. The direct health care costs of obesity in the United States. American Journal of Public Health. 1999;89(8):1194-9.

31. Druss BG, Rosenheck RA, Sledge WH. Health and disability costs of depressive illness in a major US corporation. American Journal of Psychiatry. 2000;157(8):1274-8.

32. Clarke DM, Currie KC. Depression, anxiety and their relationship with chronic diseases: a review of the epidemiology, risk and treatment evidence. Medical Journal of Australia. 2009;190(7):S54-S60.

33. Pinquart M, Duberstein PR. Depression and cancer mortality: a metaanalysis. Psychological Medicine. 2010;40(11):1797-810. 
34. Nabi H, Chastang J-F, Lefevre T, Dugravot A, Melchior M, Marmot MG, et al. Trajectories of Depressive Episodes and Hypertension Over 24 Years The Whitehall II Prospective Cohort Study. Hypertension. 2011;57(4):710-+.

35. Dortland AKBvR, Giltay EJ, van Veen T, Zitman FG, Penninx BWJH. Longitudinal Relationship of Depressive and Anxiety Symptoms With Dyslipidemia and Abdominal Obesity. Psychosomatic Medicine. 2013;75(1):83-9.

36. Van der Kooy K, van Hout H, Marwijk H, Marten H, Stehouwer C, Beekman A. Depression and the risk for cardiovascular diseases: systematic review and meta analysis. International Journal of Geriatric Psychiatry. 2007;22(7):613-26.

37. Stunkard AJ, Faith MS, Allison KC. Depression and obesity. Biological Psychiatry. 2003;54(3):330-7.

38. Penninx B, Beekman ATF, Honig A, Deeg DJH, Schoevers RA, van Eijk JTM, et al. Depression and cardiac mortality - Results from a community-based longitudinal study. Archives of General Psychiatry. 2001;58(3):221-7.

39. Goodman E, Whitaker RC. A prospective study of the role of depression in the development and persistence of adolescent obesity. Pediatrics. 2002;110(3):497-504.

40. Pine DS, Goldstein RB, Wolk S, Weissman MM. The association between childhood depression and adulthood body mass index. Pediatrics. 2001;107(5):1049-56.

41. Erickson SJ, Robinson TN, Haydel KF, Killen JD. Are overweight children unhappy? Body mass index, depressive symptoms, and overweight concerns in elementary school children. Archives of Pediatrics \& Adolescent Medicine. 2000;154(9):931-5.

42. de Wit L, Luppino F, van Straten A, Penninx B, Zitman F, Cuijpers P. Depression and obesity: A meta-analysis of community-based studies. Psychiatry Research. 2010;178(2):230-5.

43. de Wit LM, Fokkema M, van Straten A, Lamers F, Cuijpers P, Penninx BWJH. DEPRESSIVE AND ANXIETY DISORDERS AND THE ASSOCIATION WITH OBESITY, PHYSICAL, AND SOCIAL ACTIVITIES. Depression and Anxiety. 2010;27(11):1057-65.

44. Atlantis E, Baker M. Obesity effects on depression: systematic review of epidemiological studies. International Journal of Obesity. 2008;32(6):881-91.

45. John U, Meyer C, Rumpf HJ, Hapke U. Relationships of psychiatric disorders with overweight and obesity in an adult general population. Obesity Research. 2005;13(1):101-9. 
46. Baumeister $\mathrm{H}$, Harter M. Mental disorders in patients with obesity in comparison with healthy probands. International Journal of Obesity. 2007;31(7):1155-64.

47. Ohayon MM. Epidemiology of depression and its treatment in the general population. Journal of Psychiatric Research. 2007;41(3-4):207-13.

48. de Wit LM, van Straten A, van Herten M, Penninx BWJH, Cuijpers P. Depression and body mass index, a u-shaped association. Bmc Public Health. $2009 ; 9$.

49. Faith MS, Matz PE, Jorge MA. Obesity - depression associations in the population. Journal of Psychosomatic Research. 2002;53(4):935-42.

50. Scott KM, Bruffaerts R, Simon GE, Alonso J, Angermeyer M, de Girolamo G, et al. Obesity and mental disorders in the general population: results from the world mental health surveys. Int J Obes (Lond). 2008;32(1):192-200.

51. Onyike CU, Crum RM, Lee HB, Lyketsos CG, Eaton WW. Is obesity associated with major depression? Results from the Third National Health and Nutrition Examination Survey. American Journal of Epidemiology. 2003;158(12):1139-47.

52. Allison DB, Newcomer JW, Dunn AL, Blumenthal JA, Fabricatore AN, Daumit GL, et al. Obesity Among Those with Mental Disorders A National Institute of Mental Health Meeting Report. American Journal of Preventive Medicine. 2009;36(4):341-50.

53. Brumpton B, Langhammer A, Romundstad P, Chen Y, Mai XM. The associations of anxiety and depression symptoms with weight change and incident obesity: The HUNT Study. International Journal of Obesity. 2013;37(9):1268-74.

54. Koster A, van Gool CH, Kempen GIJM, Penninx BWJH, Lee JS, Rubin SM, et al. Late-Life Depressed Mood and Weight Change Contribute to the Risk of Each Other. American Journal of Geriatric Psychiatry. 2010;18(3):236-44.

55. Singh G, Jackson CA, Dobson A, Mishra GD. Bidirectional association between weight change and depression in mid-aged women: a population-based longitudinal study. International Journal of Obesity. 2014;38(4):591-6.

56. Luppino FS, de Wit LM, Bouvy PF, Stijnen T, Cuijpers P, Penninx B, et al. Overweight, Obesity, and Depression A Systematic Review and Meta-analysis of Longitudinal Studies. Archives of General Psychiatry. 2010;67(3):220-9.

57. Faith MS, Butryn M, Wadden TA, Fabricatore A, Nguyen AM, Heymsfield SB. Evidence for prospective associations among depression and obesity in population-based studies. Obesity Reviews. 2011;12(501):e438-e53. 
58. Franko DL, Striegel-Moore RH, Thompson D, Schreiber GB, Daniels SR. Does adolescent depression predict obesity in black and white young adult women? Psychological Medicine. 2005;35(10):1505-13.

59. Hasler G, Pine DS, Kleinbaum DG, Gamma A, Luckenbaugh D, Ajdacic V, et al. Depressive symptoms during childhood and adult obesity: the Zurich Cohort Study. Molecular Psychiatry. 2005;10(9):842-50.

60. Richardson LP, Davis R, Poulton R, McCauley E, Moffitt TE, Caspi A, et al. A longitudinal evaluation of adolescent depression and adult obesity. Archives of Pediatrics \& Adolescent Medicine. 2003;157(8):739-45.

61. Clum GA, Rice JC, Broussard M, Johnson CC, Webber LS. Associations between depressive symptoms, self-efficacy, eating styles, exercise and body mass index in women. Journal of Behavioral Medicine. 2014;37(4):577-86.

62. Konttinen H, Silventoinen K, Lahteenkorva SS, Mannisto S, Haukkala A. Emotional eating and physical activity self-efficacy as pathways in the association between depressive symptoms and adiposity indicators. American Journal of Clinical Nutrition. 2010;92(5):1031-9.

63. Konttinen H, Mannisto S, Sarlio-Lahteenkorva S, Silventoinen K, Haukkala A. Emotional eating, depressive symptoms and self-reported food consumption. A population-based study. Appetite. 2010;54(3):473-9.

64. Ouwens MA, van Strien T, van Leeuwe JFJ. Possible pathways between depression, emotional and external eating. A structural equation model. Appetite. 2009;53(2):245-8.

65. Angst J, Gamma A, Sellaro R, Zhang HP, Merikangas K. Toward validation of atypical depression in the community: results of the Zurich cohort study. Journal of Affective Disorders. 2002;72(2):125-38.

66. Stewart JW, McGrath PJ, Quitkin FM, Klein DF. DSM-IV Depression with Atypical Features: Is It Valid? Neuropsychopharmacology. 2009;34(13):2625-32.

67. Quitkin FM, Stewart JW, McGrath PJ, Tricamo E, Rabkin JG, Ocepekwelikson K, et al. COLUMBIA ATYPICAL DEPRESSION - A SUBGROUP OF DEPRESSIVES WITH BETTER RESPONSE TO MAOI THAN TO TRICYCLIC ANTIDEPRESSANTS OR PLACEBO. British Journal of Psychiatry. 1993;163:30-4.

68. van Gool CH, Kempen G, Penninx B, Deeg DJH, Beekman ATF, van Eijk JTM. Relationship between changes in depressive symptoms and unhealthy lifestyles in late middle aged and older persons: results from the Longitudinal Aging Study Amsterdam. Age and Ageing. 2003;32(1):81-7.

69. Strine TW, Mokdad AH, Dube SR, Balluz LS, Gonzalez O, Berry JT, et al. The association of depression and anxiety with obesity and unhealthy behaviors 
among community-dwelling US adults. General Hospital Psychiatry. 2008;30(2):127-37.

70. Lauer CJ, Wiegand M, Krieg JC. ALL-NIGHT ELECTROENCEPHALOGRAPHIC SLEEP AND CRANIAL COMPUTEDTOMOGRAPHY IN DEPRESSION - A STUDY OF UNIPOLAR AND BIPOLAR PATIENTS. European Archives of Psychiatry and Clinical Neuroscience. 1992;242(2-3):59-68.

71. Pasco JA, Jacka FN, Williams LJ, Henry MJ, Nicholson GC, Kotowicz MA, et al. Leptin in depressed women: Cross-sectional and longitudinal data from an epidemiologic study. Journal of Affective Disorders. 2008;107(1-3):221-5.

72. Vreeburg SA, Hoogendijk WJG, van Pelt J, DeRijk RH, Verhagen JCM, van Dyck R, et al. Major Depressive Disorder and Hypothalamic-Pituitary-Adrenal Axis Activity Results From a Large Cohort Study. Archives of General Psychiatry. 2009;66(6):617-26.

73. Milaneschi Y, Simonsick EM, Vogelzangs N, Strotmeyer ES, Yaffe K, Harris TB, et al. Leptin, Abdominal Obesity, and Onset of Depression in Older Men and Women. Journal of Clinical Psychiatry. 2012;73(9):1205-11.

74. Bjorntorp P. The regulation of adipose tissue distribution in humans. International Journal of Obesity. 1996;20(4):291-302.

75. Bjorntorp P. Do stress reactions cause abdominal obesity and comorbidities? Obes Rev. 2001;2(2):73-86.

76. Gangwisch JE, Malaspina D, Boden-Albala B, Heymsfield SB. Inadequate sleep as a risk factor for obesity: Analyses of the NHANES I. Sleep. 2005;28(10):1289-96.

77. Zimmermann U, Kraus T, Himmerich H, Schuld A, Pollmacher T. Epidemiology, implications and mechanisms underlying drug-induced weight gain in psychiatric patients. Journal of Psychiatric Research. 2003;37(3):193220.

78. Hasnain M, Vieweg WVR. Weight Considerations in Psychotropic Drug Prescribing and Switching. Postgraduate Medicine. 2013;125(5):117-29.

79. Vieweg WVR, Levy JR, Fredrickson SK, Chipkin SR, Beatty-Brooks M, Fernandez A, et al. Psychotropic drug considerations in depressed patients with metabolic disturbances. American Journal of Medicine. 2008;121(8):647-55.

80. Global School-based Student Health Survey (GSHS): Centers for Disease Control and Prevention (CDC). Disponível em: <Available from: http://www.cdc.gov/gshs>. Acesso em: 15 fev. 2016. 
81. Global School-based Student Health Survey (GSHS): World Health Organization (WHO). Disponível em: <http://www.who.int/chp/gshs>. Acesso em: 15 fev. 2016.

82. Joshi AV, Day D, Lubowski TJ, Ambegaonkar A. Relationship between obesity and cardiovascular risk factors: findings from a multi-state screening project in the United States. Current Medical Research and Opinion. 2005;21(11):1755-61.

83. Kenchaiah S, Evans JC, Levy D, Wilson PWF, Benjamin EJ, Larson MG, et al. Obesity and the risk of heart failure. New England Journal of Medicine. 2002;347(5):305-13.

84. Cole TJ, Lobstein T. Extended international (IOTF) body mass index cutoffs for thinness, overweight and obesity. Pediatric Obesity. 2012;7(4):284-94.

85. Chiriboga DE, Ma Y, Li W, Olendzki BC, Pagoto SL, Merriam PA, et al. Gender differences in predictors of body weight and body weight change in healthy adults. Obesity. 2008;16(1):137-45.

86. Needham BL, Crosnoe R. Overweight status and depressive symptoms during adolescence. Journal of Adolescent Health. 2005;36(1):48-55.

87. Coutinho LMS, Scazufca M, Menezes PR. Methods for estimating prevalence ratios in cross-sectional studies - Métodos para estimar razão de prevalência em estudos de corte transversal. Revista de Saúde Pública. 2008;42(6):992-8.

88. de Wit LM, van Straten A, Lamers F, Cuijpers P, Penninx BWJH. Depressive and anxiety disorders: Associated with losing or gaining weight over 2 years? Psychiatry Research. 2015;227(2-3):230-7.

89. Pine DS, Cohen P, Brook J, Coplan JD. Psychiatric symptoms in adolescence as predictors of obesity in early adulthood: A longitudinal study. American Journal of Public Health. 1997;87(8):1303-10.

90. van Strien T, van der Zwaluw CS, Engels RCME. Emotional eating in adolescents A gene (SLC6A4/5-HTT) - Depressive feelings interaction analysis. Journal of Psychiatric Research. 2010;44(15):1035-42.

91. Brener ND, McManus T, Galuska DA, Lowry R, Wechsler H. Reliability and validity of self-reported height and weight among high school students. Journal of Adolescent Health. 2003;32(4):281-7.

92. Elgar FJ, Roberts C, Tudor-Smith C, Moore L. Validity of self-reported height and weight and predictors of bias in adolescents. Journal of Adolescent Health. 2005;37(5):371-5. 
93. Sherry B, Jefferds ME, Grummer-Strawn M. Accuracy of adolescent selfreport of height and weight in assessing overweight status. Archives of Pediatrics \& Adolescent Medicine. 2007;161(12):1154-61.

94. Colman I, Wadsworth MEJ, Croudace TJ, Jones PB. Three decades of antidepressant, anxiolytic and hypnotic use in a national population birth cohort. British Journal of Psychiatry. 2006;189:156-60. 